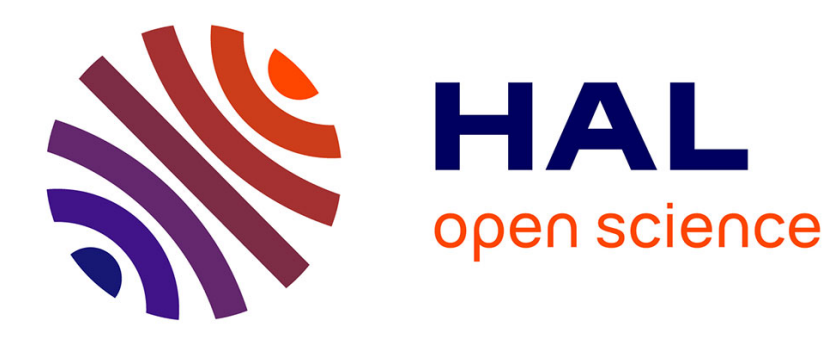

\title{
Vortex-interface interactions in two-dimensional flows
}

Daniel Fuster, Maurice Rossi

\section{To cite this version:}

Daniel Fuster, Maurice Rossi. Vortex-interface interactions in two-dimensional flows. International Journal of Multiphase Flow, 2021, 143, pp.103757. 10.1016/j.jjmultiphaseflow.2021.103757 . hal03320427

\section{HAL Id: hal-03320427 \\ https://hal.science/hal-03320427}

Submitted on 6 Oct 2021

HAL is a multi-disciplinary open access archive for the deposit and dissemination of scientific research documents, whether they are published or not. The documents may come from teaching and research institutions in France or abroad, or from public or private research centers.
L'archive ouverte pluridisciplinaire HAL, est destinée au dépôt et à la diffusion de documents scientifiques de niveau recherche, publiés ou non, émanant des établissements d'enseignement et de recherche français ou étrangers, des laboratoires publics ou privés. 


\title{
Vortex-interface interactions in two-dimensional flows
}

\author{
Daniel Fuster, Maurice Rossi \\ Sorbonne Université, Centre National de la Recherche Scientifique, UMR 7190, Institut \\ Jean Le Rond D'Alembert, F-75005 Paris, France
}

\begin{abstract}
This work focuses on the basic interactions between a vortex and an interface separating two incompressible fluids. Specifically, we investigate how vorticity produced at an interface influences the dynamics of this very same interface and how the produced vorticity engenders small scales and small droplets. A critical Weber number $W e_{c}^{(1)}$ exists discriminating the conditions upon which the interface rolls up around the vortex significantly. In addition, we show that a second critical Weber number $W e_{c}^{(2)}$ appears. For Weber numbers below this value, the dynamic response of the interface only depends slightly on the Reynolds number. For Weber numbers above $W e_{c}^{(2)}$, the vorticity layers generated by the interface itself trigger a combined RayleighTaylor and Kelvin-Helmholtz instability that leads to the appearance of extremely small structures with a characteristic size that strongly depends on the Reynolds number.
\end{abstract}

Keywords: Multiphase flow, liquid breakup, fragmentation, vorticity generation, vortex-interface interaction

\section{Introduction}

There is an extensive literature on the interaction between vorticity and an interface separating two immiscible phases. In particular, numerous experimental, numerical and theoretical analyses investigated how instabilities due to the presence of vorticity layers are modified by fluid interfaces; e.g. the Kelvin-Helmholtz instability leads to different characteristic wavelengths in the presence or not of an interface $[1,2,3]$. Reversely, the interface dynamics generates vorticity layers on the surface that subsequently alters its time evolution. To name a classical example, the Rayleigh-Taylor instability 
[4] evolves in a mushroom shape due to the subsequent roll-up of generated vorticity layers on the interface that eventually lead to the generation of ligaments and droplets $[5,6]$. In addition to problems involving the destabilization of the interface, various authors have also investigated the interactions of viscous vortices with a free surface $[7,8]$. More generally, to understand the dynamical response of multiphase flows, it seems important to characterize the mechanisms of vorticity layers formation around the interface and its consequence on the interface dynamics.

Some theoretical papers are devoted to identifying mechanisms of vorticity production by interfaces. In the limiting case of free-surface flows, $[9,10,11]$ have reported expressions of vorticity production sources at a free surface showing a law of conservation and the presence of a vortex sheet in the free boundary. More precisely in [11], the zero shear stress condition at the freesurface is transformed in a condition on vorticity while the pressure jump turns into a condition on the vorticity flux. It is precisely the vorticity flowing out of the viscous fluid domain, that accumulates inside a vortex sheet. The more general case of an interface between two viscous fluids have been tackled by $[12,13,14,15]$. To be more specific, in an series of papers, Wu and collaborators have studied in the three-dimensional case how much vorticity exists on a viscous immiscible fluid-fluid interface and how much vorticity is created from the surface [12] i.e. they provide the expressions of vorticity flux in each side of the interface. Through this approach, density, viscosity differences and surface tension become mechanisms of vorticity production. A specific discussion about the correct flux of vorticity in three-dimensional can be found in [13]. The papers $[14,15]$ discuss the same issues in twodimensional flows from the viewpoint of circulation rather than vorticity itself : indeed these authors use an extension of Kelvin's theorem of circulation conservation as their basic tool. However, these vorticity fluxes are used to understand physical problems only in a limited number of flow cases $[12,15]$. For instance, flat or axisymmetric interfaces or periodic waves are mostly considered in [15]. Note however, that an interface vortex interaction is analyzed in [15] in which the case of a free-surface is compared to the case a viscous interface. Furthermore, experimental works that quantify vorticity sheets on an interface are challenging given the small spatial scales of such layers $[16,17]$. For instance, when a drop impacts a water surface, it might generates a vortex ring. This has been related in [16] to the generation of vorticity during coaelescence caused by the relaxation of surface stresses. Al- 
ternatively, some numerical studies are available in literature : $[18,19,20,21]$ indicated the role of vorticity layers in the generation of thin structures during wave breaking; [22] have investigated the intrinsic interaction mechanisms between a bubble and turbulence; [23] reported vortex shedding due to capillary oscillations in the problem of a falling raindrop. More fundamental problems investigated the consequences of vorticity production on the recoil of a liquid ligament [24], on the pinch-off of an axisymmetric ligament [25] and on the interaction of turbulence with a free surface [26].

The present work focuses on the vorticity production on an interface in the simplest two-dimensional flow problem: the interaction of a single vortex with an interface separating two incompressible and immiscible fluids. The paper is structured as follows. Section 2 recalls some previous theoretical results about vorticity production at an interface but focusing on the case of fluids with identical dynamical viscosity. Section 3 introduces the flow problem, its non-dimensionalization and the numerical approach. Thereafter the role of various mechanisms of vorticity production (surface tension and density differences) is investigated as well as the interaction of preexisting vortex and interface. More precisely, section 4 studies the case when only surface tension is active, section 5 the case when density difference is only present. Finally the coupling of these two effects is analysed in section 6 .

\section{Vorticity production at interfaces}

This section introduces some quantities, notations and known properties of vorticity production at an interface $(I)$ characterized by a surface tension $\sigma$ separating two incompressible and immiscible fluids. Each fluid, discriminated by an index $r(r=1 \text { or } r=2)^{1}$, is of constant density $\rho^{(r)}$ and dynamical viscosity $\mu^{(r)}$. The velocity field satisfies the Navier-Stokes equation as well as the divergenceless character of velocity. In the two-dimensional plane $(x, y)$ oriented by the unit normal $\vec{e}_{z}$, the vorticity field is characterized by a unique component along $\vec{e}_{z}$ denoted by $\omega(x, y, t)$ which satisfies because

\footnotetext{
${ }^{1}$ Whenever necessary, the notation $Q^{(r)}$ is explicitly used to stand for the quantity $Q$ in phase $r=1$ or $r=2$. Notations $[[Q]]$ and $Q_{m}$ respectively stand for the difference $\left(Q^{(1)}-Q^{(2)}\right)$ and the mean value $\left(Q^{(1)}+Q^{(2)}\right) / 2$ at a point of the interface.
} 
of Navier-Stokes equation

$$
\frac{D \omega^{(r)}}{D t}=-\nabla \cdot \vec{J}^{(r)}, \quad \vec{J}^{(r)} \equiv-\nu^{(r)} \vec{\nabla} \omega^{(r)}
$$

where $D / D t$ is the Lagrangian time derivative and $\nu^{(r)}=\mu^{(r)} / \rho^{(r)}$ is the kinematic viscosity.

In the $x-y$ plane, consider a Lagrangian surface $A$ delimited by a closed Lagrangian curve $(C)$. One introduces on the Lagrangian curve $(C)$, vector $\vec{n}$ to be the outgoing unit normal vector. The conservation of circulation $\Gamma$ i.e. the flux of vorticity over a Lagrangian surface $A$ delimited by a closed Lagrangian curve $(C)$ is generalized for a two-phase flow (see $[12,14])$

$$
\frac{\mathrm{d} \Gamma}{\mathrm{d} t}=-\int_{C} J_{j} n_{j} \mathrm{~d} s_{c}+\int_{I} \Sigma \mathrm{d} s, \quad \Gamma \equiv \int_{A} \omega \mathrm{d} x \mathrm{~d} y
$$

The first r.h.s. integral is a classical viscous diffusion term through a boundary $(C)$ and the second integral is a supplementary term corresponding to the vorticity sources located at the interface $(I)$. Variable $s_{c}$ denotes the curvilinear coordinates defined on $(C)$ that increases in the direction of the tangent vector $\vec{t}=\vec{e}_{z} \times \vec{n}$ which prescribes the orientation of curve $(C)$. Variable $s$ denotes the curvilinear coordinates defined on $(I)$ that increases in the direction of $\vec{t}^{1 \rightarrow 2}=\vec{e}_{z} \times \vec{n}^{1 \rightarrow 2}$ where unit normal vector $\vec{n}^{1 \rightarrow 2}$ is directed from phase 1 to 2 . The tangential stress is known to be continuous across interface (I) but the normal stress follows the Young -Laplace law

$$
[[p]]=-\sigma \kappa+2[[\mu]] \kappa n_{j}^{1 \rightarrow 2} u_{j}-2[[\mu]] t_{i}^{1 \rightarrow 2} \partial_{i}\left(t_{j}^{1 \rightarrow 2} u_{j}\right),
$$

where the interface curvature $\kappa$ is positive when the centre of curvature lies in phase 2. The source term is known to be expressed as

$$
\Sigma=\vec{t} \cdot \vec{\nabla}\left[\left[\frac{p}{\rho}\right]\right]
$$

or using the mean pressure $p_{m}=\left(p_{1}+p_{2}\right) / 2[27]$

$$
\Sigma=\frac{1}{2}\left(\frac{1}{\rho_{1}}+\frac{1}{\rho_{2}}\right) \vec{t} \cdot \vec{\nabla}[[p]]+\left[\left[\frac{1}{\rho}\right]\right] \vec{t} \cdot \vec{\nabla} p_{m} .
$$

Across interface $(I)$, vorticity is generated to comply with the no-slip condition imposed on the velocity field at an interface. Otherwise stated, this 
production is related to the boundary layer present close to an interface. The sources of vorticity being always generated by the scalar products of $\vec{t}$ with a gradient, when interface $(I)$ is a closed contour (e.g. phase 1 included inside phase 2 ), the total production of vorticity $\int_{(I)} \Sigma \mathrm{d} s$ is null: negative and positive production are canceling each other. The same analysis provides as well the source $\Sigma_{1}$ in phase 1 and the source $\Sigma_{2}$ in phase 2

$$
\begin{aligned}
& \Sigma_{1}=\frac{\vec{t}}{2 \rho^{(1)}} \cdot \vec{\nabla}[[p]]+\frac{\vec{t}}{\rho^{(1)}} \cdot \vec{\nabla} p_{m}+\vec{t} \cdot \frac{D \vec{u}}{D t}, \\
& \Sigma_{2}=\frac{\vec{t}}{2 \rho^{(2)}} \cdot \vec{\nabla}[[p]]-\frac{\vec{t}}{\rho^{(2)}} \cdot \vec{\nabla} p_{m}-\vec{t} \cdot \frac{D \vec{u}}{D t}
\end{aligned}
$$

Note that for any quantity $Q$ defined on on boundary $(I), \partial Q / \partial s \equiv \vec{t} \cdot \vec{\nabla} Q$.

In the present work, we assume that dynamical viscosity is identical for both fluids i.e. $[[\mu]]=0$. While continuity of velocity across the interface is always ensured when the two dynamical viscosities are different or not, the condition $[[\mu]]=0$ implies that the velocity gradient tensor itself is also continuous across the interface

$$
\left[\left[\omega_{i}\right]\right]=0, \quad\left[\left[\partial_{i} u_{j}\right]\right]=0 \quad \text { on an interface with }[[\mu]]=0 .
$$

Vorticity is hence continuous but a vorticity flux is nonetheless present. There are several reasons for this assumption. First there exists many vorticity sources and one paper is not enough to cover all of them. We choose to investigate density and surface tension effects only. Second this facilitates the comparisons between mono-phasic flow and two-phase flow. For each problem, we analyze how the vortex-interface system evolves differently in time with respect to the mono-phasic case by producing small scales or by changing the global flow. To do so we provide identical initial conditions for the velocity field in the mono-phasic and multi-phasic flows. This is possible only when $[[\mu]]=0$ : boundary conditions at the interface for the two-phase flow whatever its position might be, are then automatically satisfied at initial time if satisfied in the mono-phasic flow. Third it is much more difficult to ensure convergence of the numerical results for the case for $[[\mu]] \neq 0$ because of viscous normal constraints or equivalentlly the Young -Laplace law is simpler since only the first term in equation (3) remains. In any case, assuming $[[\mu]]=0$ is certainly a realistic assumption for liquid/liquid systems represented by the small density difference cases considered here. Finally 
the vorticity source due to dynamic viscosity differences can be shown to be inversely proportional to the Reynolds number [27] and therefore it is safe to assume that its contribution is negligible compared to the vorticity production due to baroclinic effects.

Let us make some subsidiary remarks about the case $\rho_{1}<<\rho_{2}$. In such an instance, $[[v]] \sim 1 / \rho^{(1)}, v_{m} \sim 1 /\left(2 \rho^{(1)}\right)$ and $\Sigma$ depends on the lighter fluid

$$
\Sigma^{(1)} \approx \Sigma+\vec{t}^{1 \rightarrow 2} \cdot \frac{D \vec{u}}{D t}, \quad \Sigma^{(2)} \approx-\vec{t}^{1 \rightarrow 2} \cdot \frac{D \vec{u}}{D t}
$$

When the "tangential acceleration" term $\vec{t} \cdot \frac{D \vec{u}}{D t}$ is small compared to the production $\Sigma$, most of flux of vorticity is taking place inside the lighter fluid (here fluid 1).

$$
\Sigma^{(1)} \approx \Sigma \quad\left|\Sigma^{(2)}\right|<<\left|\Sigma^{(1)}\right|
$$

By contrast, when the acceleration term $\vec{t}^{1 \rightarrow 2} \cdot(D \vec{u} / D t)$ is large compared to $\Sigma$, the two fluxes of vorticity are almost opposite $\Sigma^{(1)} \approx-\Sigma^{(2)}$.

\section{Problem description and numerical methods}

In this work, the interaction between vorticity and an interface with a surface tension $\sigma$ is analyzed in its simplest form: a single vortex interacts with an interface and the regions of initial vorticity and vorticity production are well separated. More precisely an initial flat interface separates fluid 1 located at $x<d_{0}$ from fluid 2 located $x>d_{0}$. This interface interacts with a LambOseen vortex centered at $x=0$, characterized by a circulation $\Gamma_{0}$ and a core size smaller than $d_{0}$ so that the interface is almost deprived of vorticity. Using the dimensional quantities $U_{0}=\Gamma_{0} /\left(2 \pi d_{0}\right)$ and $L_{0}=d_{0}$, together with the average density $\rho_{m}=\left(\rho^{(1)}+\rho^{(2)}\right) / 2$, the initial velocity is expressed in dimensionless units and polar coordinates by

$$
u_{\theta}=\frac{1-\exp \left(-\left(r / a_{0}\right)^{2}\right)}{r}, \quad \omega_{z}=\frac{2}{a_{0}^{2}} \exp \left(-\left(r / a_{0}\right)^{2}\right)
$$

with $a_{0}$ the dimensionless initial core size which is one of the four dimensionless numbers governing the dynamics with the Reynolds number, Weber number

$$
R e \equiv \frac{\rho_{m} \Gamma_{0}}{2 \pi \mu}, \quad W e \equiv \frac{\rho_{m} \Gamma_{0}^{2}}{(2 \pi)^{2} d_{0} \sigma}
$$


and density ratio

$$
r_{\rho} \equiv \frac{\rho^{(2)}}{\rho^{(1)}}=\frac{\nu^{(1)}}{\nu^{(2)}} .
$$

The dimensionless form, the source $\Sigma$ becomes

$$
\Sigma=\frac{r_{\rho}+1}{2 r_{\rho}}\left(\left(r_{\rho}-1\right) \frac{\partial p_{m}}{\partial s}-\frac{\left(r_{\rho}+1\right)}{2} \frac{1}{W e} \frac{\partial \kappa}{\partial s}\right)
$$

In the classical monophasic case $\left(r_{\rho}=1\right.$ and $\left.W e=\infty\right)$, when there is no vorticity $\operatorname{source}(\Sigma=0)$, the vortex evolves as a Lamb-Oseen vortex and the interface evolves as a passive scalar (see Appendix A for details). In the cases $r_{\rho} \neq 1$ or $W e \neq \infty$, the vortex evolves differently from a pure Lamb-Oseen vortex and the interface is no longer passive.

In what follows we consider two core sizes $a_{0}=1$ and $a_{0}=0.1$ which enable to investigate the role of pre-existing vorticity on the initial interface: the initial vorticity is mainly located in fluid 1 and it is weak near the interface for $a_{0}=1$ or almost zero for $a_{0}=0.1$.

In the present work, we solve the Navier-Stokes equations in both fluids in the dimensionless form using pressure and velocity as primary variables

$$
\frac{\rho^{(r)}}{\rho_{m}}\left[\frac{\partial u_{i}^{(r)}}{\partial t}+\left(u_{j}^{(r)} \partial_{j}\right) u_{i}^{(r)}\right]=\partial_{j} p^{(r)}+\frac{1}{R e} \partial_{j} \partial_{j} u_{i}^{(r)}, \quad \partial_{i} u_{i}^{(r)}=0,
$$

with

$$
\frac{\rho^{(1)}}{\rho_{m}}=\frac{2}{\left(1+r_{\rho}\right)}, \quad \frac{\rho^{(2)}}{\rho_{m}}=\frac{2 r_{\rho}}{\left(1+r_{\rho}\right)}
$$

The dimensionless normal stress condition becomes

$$
[[p]]=-\frac{\kappa}{W e}
$$

In particular we use Basilisk [28], which have been widely used and tested for the computation of incompressible two-phase flow problems [29, 30, 31] and accurate methods for curvature computation based on height functions can be readily used [32]. The code solves the Navier-Stokes equations by using the one fluid model, where the interface is represented by a fast but continuous 
change of the fluid properties, density and viscosity, as a function of the volume fraction $c$ that is advected using the Volume of Fluid method proposed by [33]. The method combines a modified version of the Bell-Colella-Glaz for advection, an implicit method to treat viscous terms and a projection step to ensure that the field is divergence free. The dimensionless domain is given by a square box $100 \times 100$ with impenetrability and slip wall conditions on the domain boundary. An adaptive grid is required in this case to capture well the dynamics of the tip. The maximum resolution is varied from $\Delta_{x}=310^{-3}$ for simulations at high Weber numbers to $\Delta_{x}=1.210^{-2}$ for simulations at $W e$ below 100. Such high resolutions are required to accurately capture the flow field originated as a consequence of vorticity production. Independent numerical tests have indicated that to resolve the vorticity production requires $\Delta \leq 10-100 l_{\mu}$, with $l_{\mu}=L_{c} O h^{2}$ a characteristic length that depends on the the Ohnersorge number $\left(O h=\sqrt{W e} R e=\frac{\mu}{\sqrt{\rho_{m} \sigma L_{0}}}\right)$ and $L_{c}$ the characteristic length of the problem. This condition can be also written as

$\frac{\Delta}{L_{c}} \leq 10-100 \frac{W e}{R e^{2}}$. The simulations reported in this manuscript are carried out with extremely high resolution $\frac{\Delta}{L_{c}}=510^{-4}$ in order to correctly resolve the vorticity production for $W e \geq 5$.

\section{A vortex near an interface with surface tension only.}

In this section, we focus on the simplest possible case: surface tension is present but without any density difference i.e. we set $r_{\rho}=1$. This case, called problem $P_{1}$, is the limit when density differences are negligible. For large Weber numbers, the interface dynamics is mainly changed near the tip (figure 1) where the curvature is stronger. The curvature at the tip is actually reduced compared to the passive interface and, even for large Weber numbers $\left(W e=10^{3}\right.$ and $W e=10^{2}$ in figure 1 ), a rim is observed. A rim appears on the ligament, when a constriction of the ligament is followed by an enlargement. As surface tension is increased, this rim becomes larger (compare $W e=10^{3}$ and $W e=10^{2}$ in figure 1 ). In addition, there exists a critical range of Weber number such that for $W e$ below this range, surface tension prevents any roll-up (see $W e=10$ in figure 1 ). In this case, a vorticity layer can be detached from the interface tip thus leading to vortex shedding, producing a dipole and capillary waves.

The above dynamics can be understood by analyzing vorticity production. 

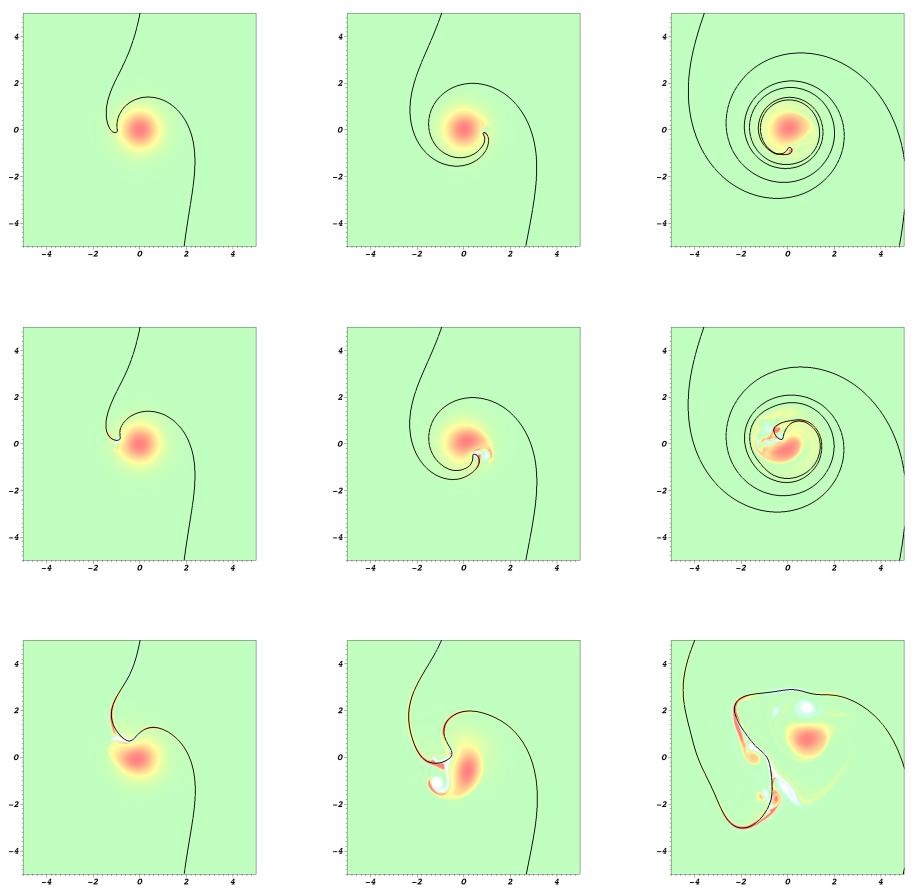

Figure 1: Problem $P_{1}$ and $R e=1000$. Simulations for times $t=5,10,30$ at $W e=10^{3}$ (top) $W e=10^{2}$ (middle) $W e=10$ (bottom).
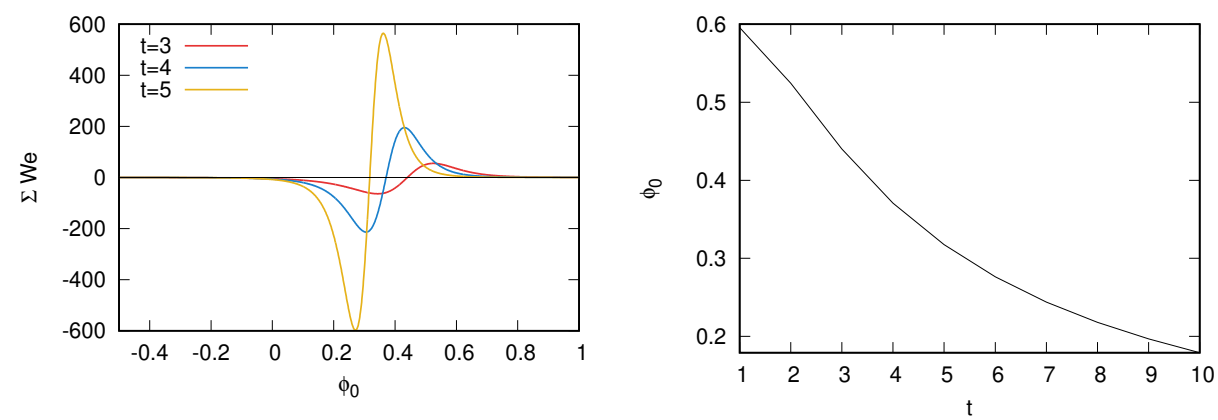

Figure 2: Problem $P_{1}$. (left) Rescaled source $W e \Sigma$ of vorticity as a function of $\phi_{0}$ using the analytical spiral at time $t=3,4,5$ with $a_{0}=1$. (right) Position $\phi_{0}(t)$ of maximum curvature as the function of time for the analytical spiral. 

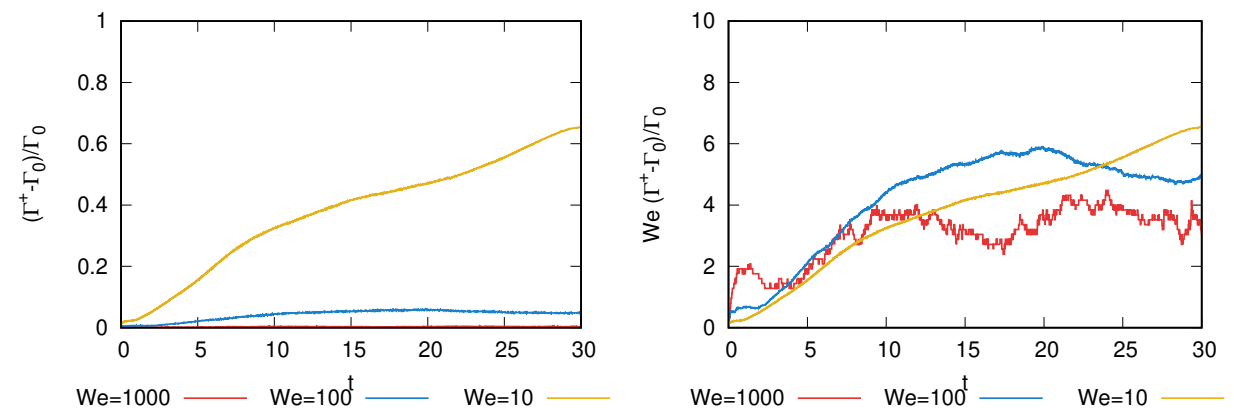

Figure 3: Problem $P_{1}$ for $R e=1000$. Left: Temporal evolution of the relative increment $\left(\Gamma^{+}-2 \pi\right) / 2 \pi$ of the integral of positive vorticity with respect to the Lamb-Oseen case. Right: figure rescaled by multiplying by Weber number.

For problem $P_{1}$, the source (14) is proportional to the gradient of curvature along the interface

$$
\Sigma=-\frac{1}{W e} \frac{\partial \kappa}{\partial s} .
$$

This term may be evaluated in a crude way by assuming the interface to be close to the passive spiral and by computing (18) along this spiral (in these analytical computations, the viscous diffusion in $a_{v}(t)$ is discarded i.e. $\left.a_{v}(t)=a_{0}\right)$. This is surely correct at short times. The source at the interface (figure 2 left) is localized around positive values on the upper part (above the maximum curvature point where the source goes to zero) and negative on the lower part. The points of highest positive or negative production are nearby the maximum curvature point, which is shifting towards $\phi_{0}=0$ with time (figure 2 right). This is indeed observed in figure 1: the generation of vorticity is mainly concentrated near high curvature position so it is localized in certain points of the interface forming a sort of dipole. In the rough approximation using passive spiral, the added circulation must be proportional to surface tension i.e inversely proportional to $W e$. This remains valid for NavierStokes simulations : on the left figure 3, one displays the relative increment $\left(\Gamma^{+}-2 \pi\right) / 2 \pi$ of the integral of positive vorticity removing the initial vortex circulation $2 \pi$. On the right figure, the same increase is shown to scale with Weber number.

As surface tension is increased, the amount of vorticity generated by the interface increases as well as the rim size (compared $W e=10^{3}$ and $W e=10^{2}$ 

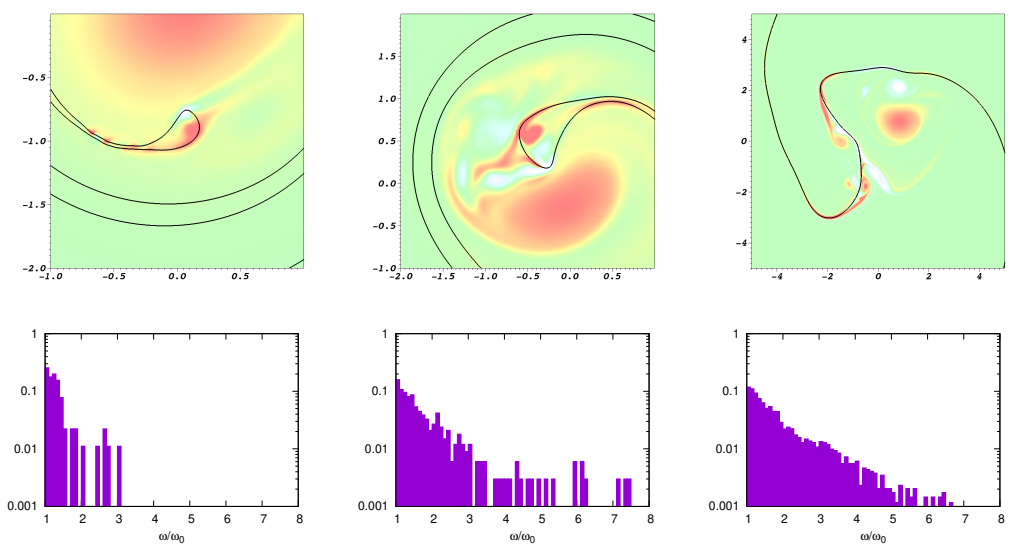

Figure 4: Problem $P_{1}$ for $R e=1000$ and $a_{0}=1$. Top: Zoomed view of ligaments and vorticity fields around them (from left to right) at $W e=1000,100,10$. Bottom: probability density functions of $\omega / \omega_{0}$ for $\omega>\omega_{0}$.

in Figure 1). The vorticity produced at the interface may become more intense than $\omega_{0}=\frac{2}{a_{v}^{2}}$ the peak vorticity of the initial vortex. In order to quantify this effect, one computes the histogram of cells where vorticity is larger than $\omega_{0}$ and normalizes these histograms by dividing by the total number of such cells. This provides an approximation of the probability density function (pdf) of values of $\omega / \omega_{0}$ greater than unity. Most values of vorticity are relatively close to $\omega / \omega_{0}=1$ for $W e=10^{3}$ (see figure 4 ). When the Weber number $W e$ is decreased, there are more cells with larger vorticity values. However the differences between $W e=10$ and $W e=100$ becomes less significant. Similar structures of the vorticity field lead to comparable values of the nondimensional vorticity predicted. Of course the size of the structures are larger as the Weber number $W e$ decreases but the decrease of curvature is compensated by the increase of surface tension thus yielding an approximately constant value of the peak vorticity produced. The vorticity produced at interface generates itself a velocity field that is estimated in a rough manner as follows. First one computes a vorticity field $\tilde{\omega}$ filtered from the total vorticity field $\omega$ by removing the vorticity inside the deformed vortex (for times $t=2$ and $t=7$ and case $a_{v}=0.1$ shown in figure 5 , this is doable since the vortex remains well-separated from the vorticity previously generated and shed by the interface). Second the streamfunction $\psi$ generated 

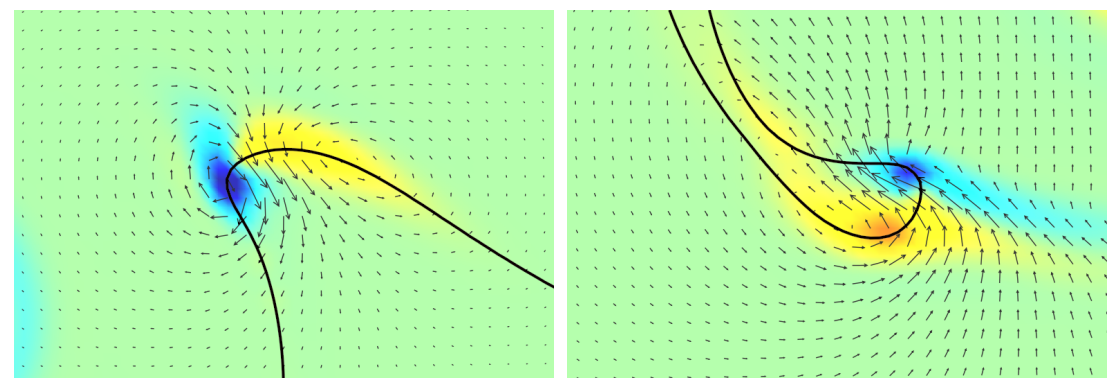

Figure 5: Problem $P_{1}$ for $R e=1000, W e=100$ and $a_{0}=0.1$ : Velocity field at (left) $t=2$ and (right) $t=7$ obtained from the filtered vorticity.
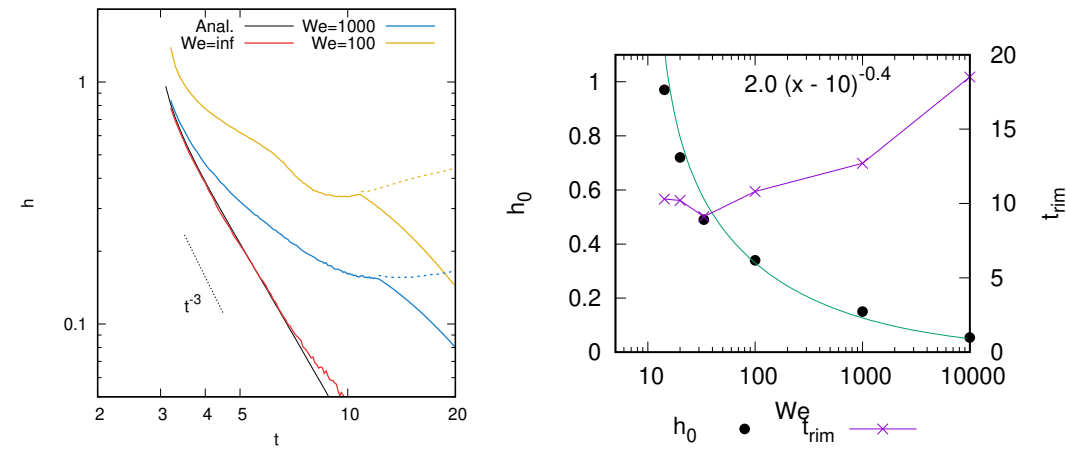

Figure 6: Problem $P_{1}$ for $R e=1000, a_{0}=1$ and various Weber numbers. (Left) Temporal evolution of $h_{\text {min }}(t)$ (continuous line) and $h_{\max }(t)$ (dashed line). (Right, dots and green line) ligament thickness $h_{0}$ and (crosses and violet line) rim formation time $t_{\text {rim }}$ as a function of Weber number $W e$.

by a filtered vorticity field $\tilde{\omega}$ is obtained by solving

$$
\nabla^{2} \psi=-\tilde{\omega}
$$

which then yields the velocity field through $u_{x}=\partial_{y} \psi, u_{y}=-\partial_{x} \psi$. Figure 5 shows that this velocity field is rather dipolar. This field is at the origin of the rim formation ( $W e=10^{3}$ and $W e=10^{2}$ in Figure 1).

In Appendix B, we define in a more quantitative way, the width $h_{\min }(t)$ of a ligament as well as the time $t_{\text {rim }}$ when the rim appears. The procedure based on the simulation results is also presented. For $t>t_{\text {rim }}$, one also introduces the rim size $h_{\max }(t)$ as the maximum of the distance function with 

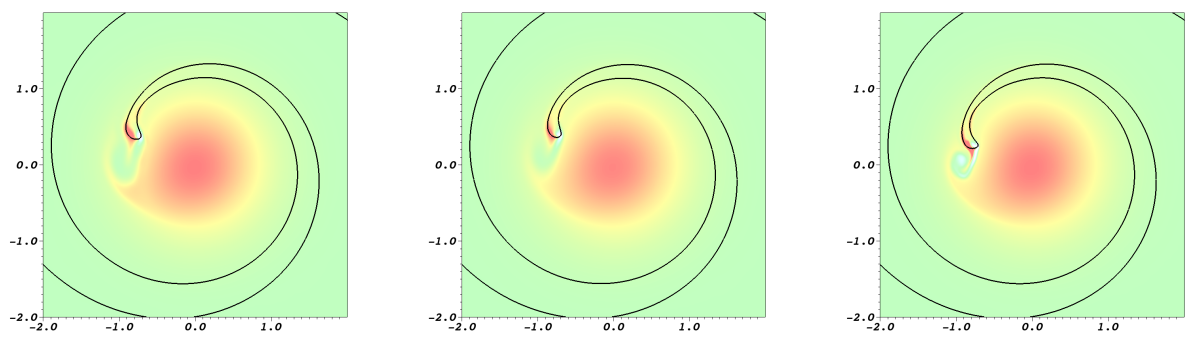

Figure 7: Problem $P_{1}$ for $a_{0}=1$ and $W e=10^{3}$ : Influence of Reynolds Vorticity field and interface at time $t=15$ for (left) $R e=10^{3}$, (middle) $R e=210^{3}$ and (right) $R e=10^{4}$.

respect to the interface inside the rim. Figure 6(left) displays the temporal evolution of the width $h_{\min }(t)$ of a ligament (continuous line) for various Weber numbers. Before the rim forms, $h_{\text {min }}(t)$ decreases still algebraically in time $h_{\min }(t) \propto 1 / t^{n}$ but the exponent $n$ is less than 3. For $t>t_{\text {rim }}$, i.e. once the rim is formed, $h_{\min }(t)$ is closer to $1 / t^{3}$ and the rim size $h_{\max }(t)$ (dashed line) slightly increases with respect to its first value at $t_{\text {rim }}$ that is $h_{0} \equiv h_{\text {min }}\left(t_{\text {rim }}\right)$. The thickness $h_{0}$ and the formation time $t_{\text {rim }}$ are both functions of Weber number (see figure 6). As expected, the characteristic size of the rim decreases when increasing the Weber number while the formation time increases. The characteristic size $h_{0}$ is also used to numerically obtain the critical $W e$ below which no rim appears during the rolling process: actually it is a fuzzy but narrow region around $W e_{c} \approx 14$.

Finally, note that the dynamics of the interface does not change for a large range of Reynolds numbers irrespective of the value of the Weber number. In particular Figure 7 displays the dependence of the vorticity field for $W e=1000$ for $R e=10^{3}, 2.10^{3}, 10^{4}$ : in problem $P_{1}$, the Reynolds number does not influence the interface shape significantly even for relatively long times. A discussion on the influence of the Reynolds number on the structures observed is postponed to the last section of the manuscript. 

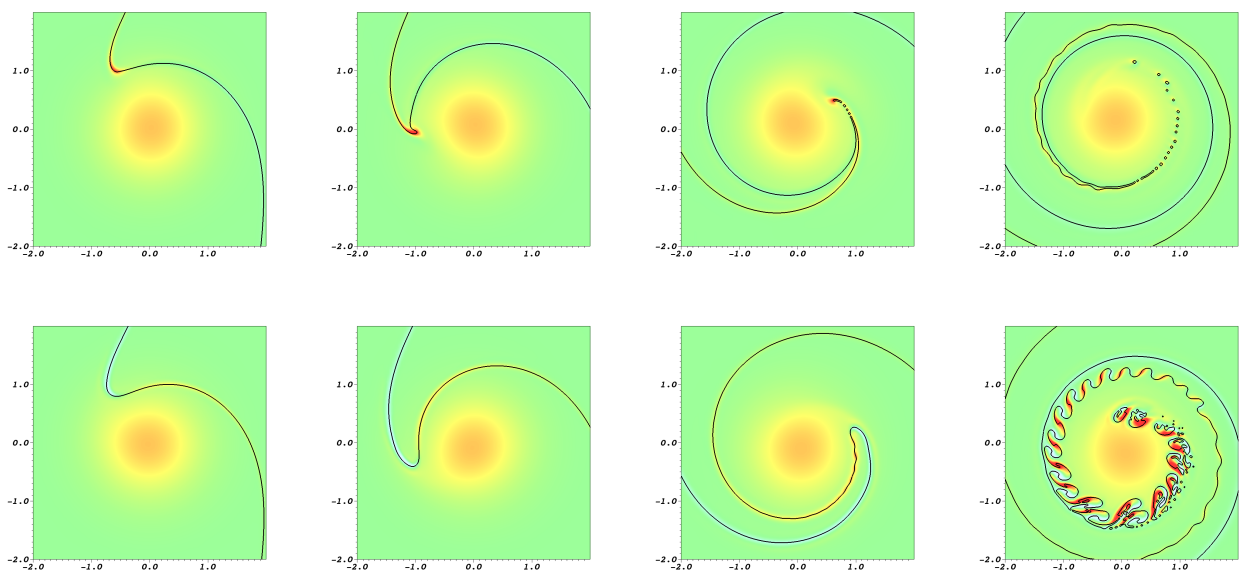

Figure 8: Problem $P_{2}$ for (top) $r_{\rho}=1.2$ or (bottom) $r_{\rho}=0.8$ and $R e=10^{3}, a_{0}=1$ : vorticity field and interfaces at times $t=3,5,10,20$. Red color means positive vorticity, blue negative vorticity.

\section{A vortex near a density jump only.}

Let us analyze the complementary case to problem $P_{1}$ called problem $P_{2}$ : density difference is present $\left(r_{\rho} \neq 1\right)$ but surface tension is set to zero. The interface (figure 8) starts to roll up under the action of the pre-existing vortex but, unlike problem $P_{1}$, the tip is rapidly modified compared to the passive case even for $r_{\rho}$ close to one. In that instance, the source term generates a vorticity layer over the interface instead of a localized dipole near the high curvature points. This can be accounted for by the dimensionless vorticity source (14) : when density differences is the only mechanism, it reduces to the baroclinic term

$$
\Sigma=\frac{r_{\rho}^{2}-1}{2 r_{\rho}} \frac{\partial p_{m}}{\partial s} .
$$

Consider the rough approximation in which the observed spiral is assumed close to the passive one and the pressure field $p_{m}$ to be mainly due to the unperturbed Lamb-Oseen vortex. This approximation evaluates the vorticity production along the spiral

$$
\frac{\partial p_{m}}{\partial s}=\frac{\partial p_{m}}{\partial r} \frac{\partial r}{\partial s}=\frac{u_{\theta}^{2}}{r} \frac{\partial r}{\partial s}=\frac{u_{\theta}^{2}}{r} \frac{\partial r}{\partial \phi_{0}} \frac{1}{\frac{\partial s}{\partial \phi_{0}}}
$$



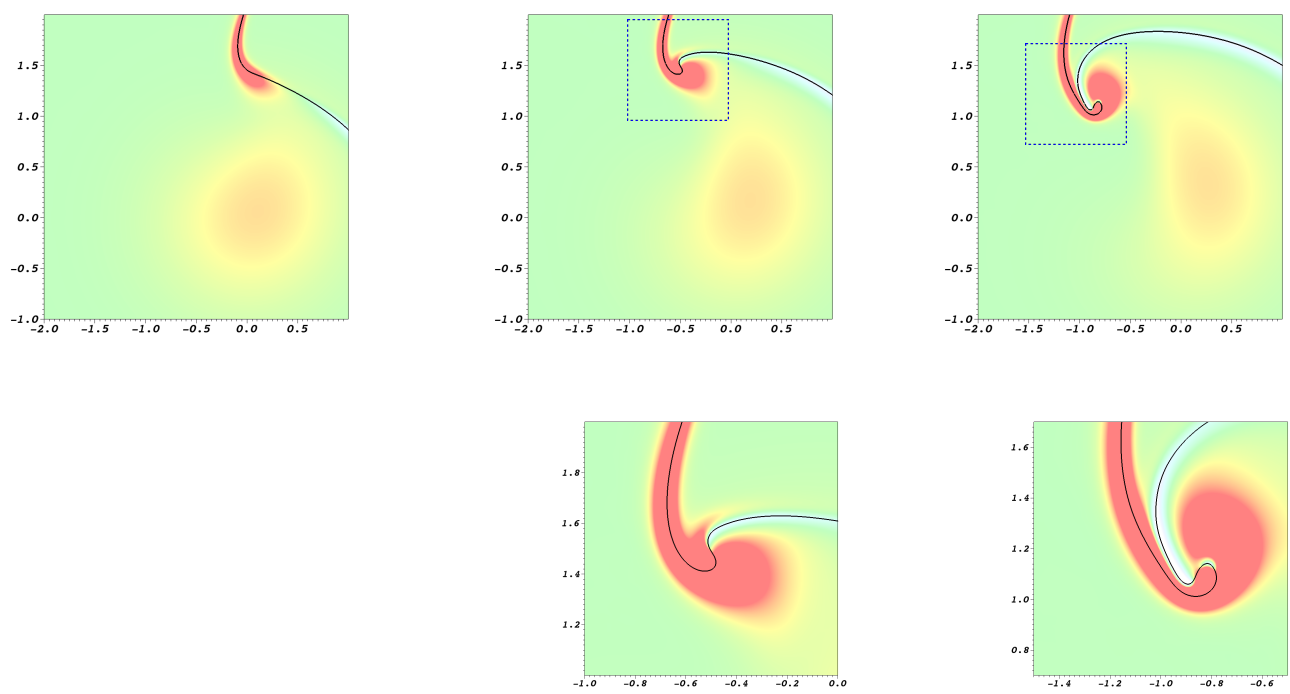

Figure 9: Problem $P_{2}$ for $r_{\rho}=5, R e=1000$ and $a_{0}=1$. (top) Vorticity fields at $t=2,3,4$. (bottom) Zoomed view of the tip at $t=3,4$.

where quantities in equation (21) are computed in Appendix A. Contrary to problem $P_{1}$, the source term (figure 10) is not concentrated near high curvature points but it is much less localized over the interface thus generating a layer. In addition this term changes sign at a point given by $\phi_{0}=0$ which is always shifted from the position of the ligament tip.

\subsection{Ligament dynamics and the following instability for $r_{\rho}>1$}

For $r_{\rho}>1$, the dynamics of the ligament tip are quickly modified with respect to the pure passive tracer and small structures at the ligament tip appear (see for example figure (9)). The value of the vorticity source approximated by Eq. (21) reveals that vorticity production at the interface is positive on the upper part $\left(\phi_{0}>0\right)$ and negative on the lower part $\left(\phi_{0}<0\right)$. Within the same theoretical model, the rescaled positive production $\frac{2 r_{\rho}}{r_{\rho}^{2}-1} \int_{\Sigma>0} \Sigma d s$ is larger than the rescaled negative production $\frac{2 r_{\rho}}{r_{\rho}^{2}-1} \int_{\Sigma<0} \Sigma d s$ (see figure 10 

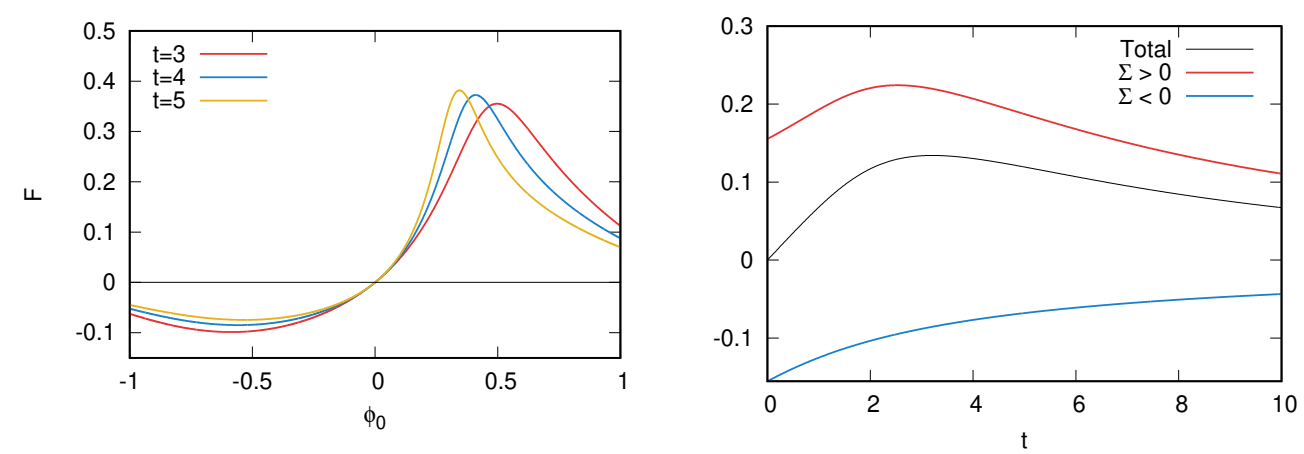

Figure 10: Problem $P_{2}$ in the passive spiral approximation: (Left) Renormalized vorticity source $F=\frac{2 r_{\rho}}{r_{\rho}^{2}-1} \Sigma$ as a function of $\phi_{0}$ in the range $[-1,1]$ at times $t=3,4,5$. (Right) For $r_{\rho}>1$, rescaled vorticity production $\frac{2 r_{\rho}}{r_{\rho}^{2}-1} \int_{(I)} \Sigma d s$ (black line), positive production $\frac{2 r_{\rho}}{r_{\rho}^{2}-1} \int_{\Sigma>0} \Sigma d s$ (red line) and negative production $\frac{r_{\rho}^{2}-1}{2 r_{\rho}} \int_{\Sigma<0} \Sigma d s$ (blue line).

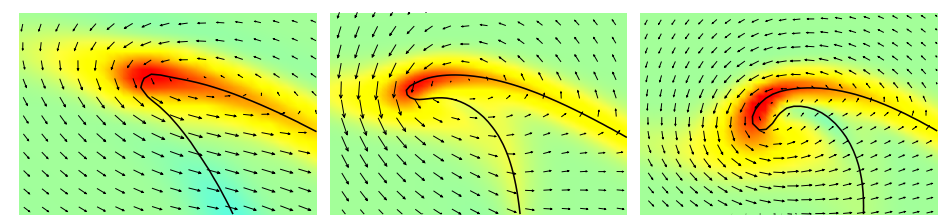

Figure 11: Problem $P_{2}$ for density ratios $r_{\rho}=1.2,2,5$ (from left to right) and $R e=1000$, $a_{0}=0.1$. Velocity field obtained using the filtered vorticity field at $t=2.4$. Color represents the vorticity field.

(right) as a function of time). The production of vorticity for $r_{\rho}>1$ and $\phi_{0}>0$ is positive and its contribution dominates the overall production (figure 10). This situation is thus similar to the starting roll-up of a plane vortex sheet, but the vorticity sheet here is generated through surface conditions.

Figure 11 displays the velocity field produced by the interface vorticity obtained using Eq. 19. This extra velocity field intensifies the action of the pre-existing vortex velocity field leading to an increased stretching of the ligament and thus its thinning compared to the passive case (compare figure A.27 and figure 8). When vorticity generated at the interface becomes large enough, the velocity generated by such an extra vorticity becomes larger than the velocity induced by the pre-existing vortex. Thereafter, the generated 

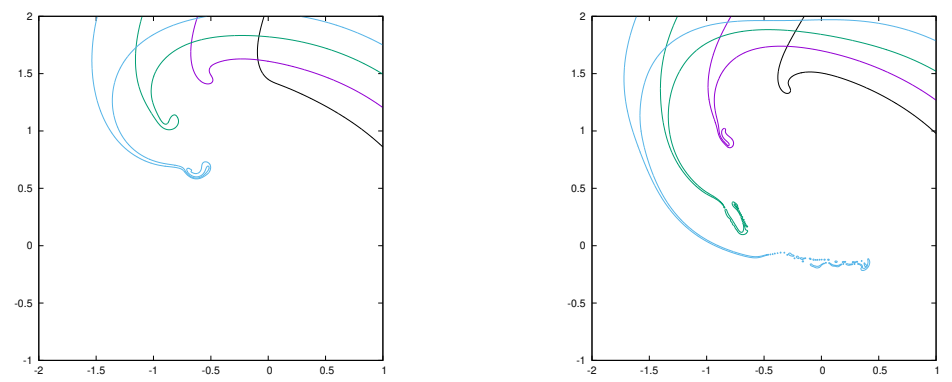

Figure 12: Problem $P_{2}$ for $r_{\rho}=5, R e=1000$ and (left) $a_{0}=1$ or (right) $a_{0}=0.1$ : The interface zoomed near the tip at $t=2,3,4,5$.
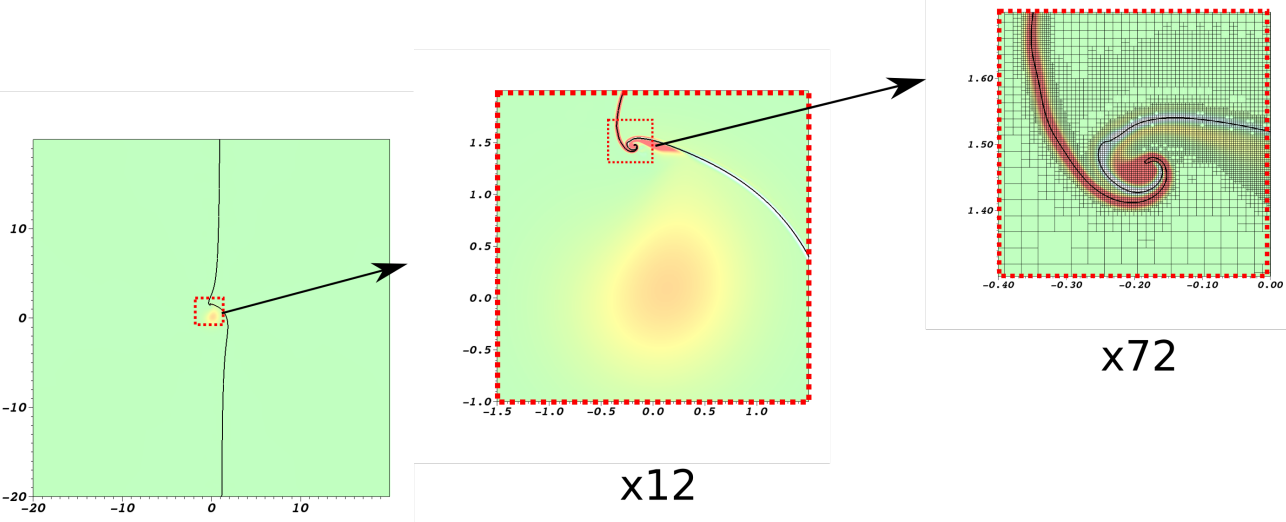

Figure 13: Problem $P_{2}$ for $r_{\rho}=5, R e=10^{4}, a_{0}=0.1$. Vorticity and interface contours at $t=2.4$. (Left) Global view, (middle) zoomed view around the vortex core (right) zoomed view at the ligament tip and grid 

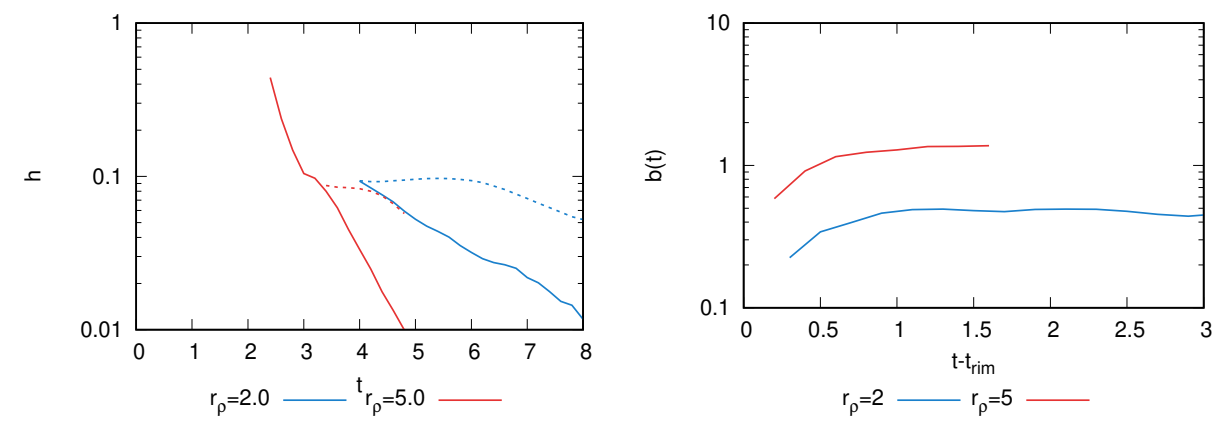

Figure 14: Problem $P_{2}$ for various density differences $r_{\rho}$ and $R e=1000$ and $a_{0}=1$ (Left) Temporal evolution of $h_{\min }(t)$ (continuous line) and $h_{\max }(t)$ (dashed line); (Right) Rate $b(t) \equiv \frac{-1}{t-t_{\text {rim }}} \log \left(\frac{h_{\min }(t)}{h_{0}}\right)$ as a function of time after rim formation.

vorticity rolls up and is shed over the tip to create a new vortex (figure 9). In turn, this vortex influences the dynamic response of the interface initiating fluid fragmentation near the tip that eventually lead to small numerical fragments of fluid (figure 12 for $r_{\rho}=5$ at $t=5$ ). Contrary to problem $P_{1}$, the ligament generated for $r_{\rho}>1$ does rapidly break, this behaviour being accentuated for increasing $r_{\rho}$ and modified by the presence of initial vorticity on the surface (compare the evolution for $a_{0}=1$ and $a_{0}=0.1$ in figure 12). In particular in the case of $a_{0}=1$ we observe larger structures than those observed when the vortex core size is smaller than the distance to the interface. For density ratios above $r_{\rho}>5$, this layer detaches and generates a new vortex around which the interface rolls up. This leads to the appearance of extremely small structures that demand high resolution around the tip (Figure 13).

The temporal evolution of the ligament thickness $h_{\min }$ (figure 14 left), shows that the necking process is no longer algebraic but better fitted by an exponential law where $b(t) \equiv \frac{-1}{t-t_{\text {rim }}} \log \left(\frac{h_{\min }(t)}{h_{0}}\right)$ reaches a plateau $b_{0}$ which is larger for larger $r_{\rho}$ (see Figure 14(right)). The evolution of the ligament dynamic thickness is then described by

$$
h_{\text {min }}(t) \approx h_{0} \exp \left(-b_{0}\left(t-t_{\text {rim }}\right)\right) .
$$

As mentioned above, an extra velocity field due to the vortex layer intensifies 

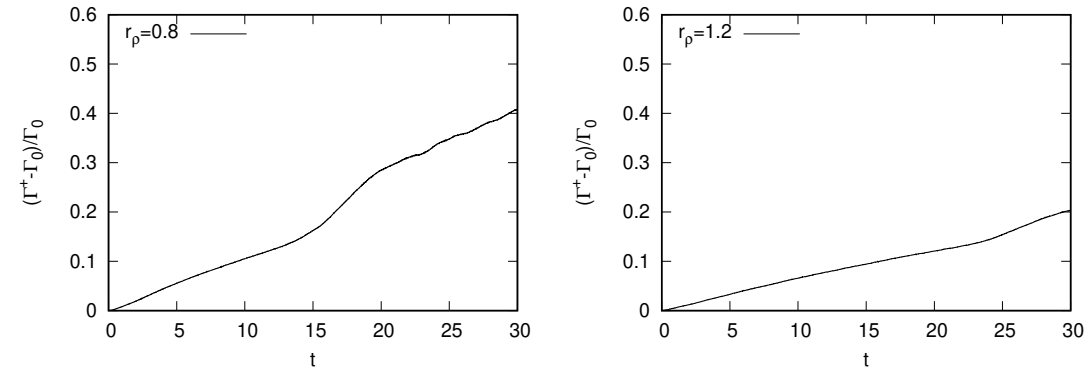

Figure 15: Problem $P_{2}$ for (left) $r_{\rho}=0.8$, (right) $r_{\rho}=1.2$ and $R e=10^{3}, a_{0}=1$ : Evolution of the circulation related to the regions of positive vorticity field.
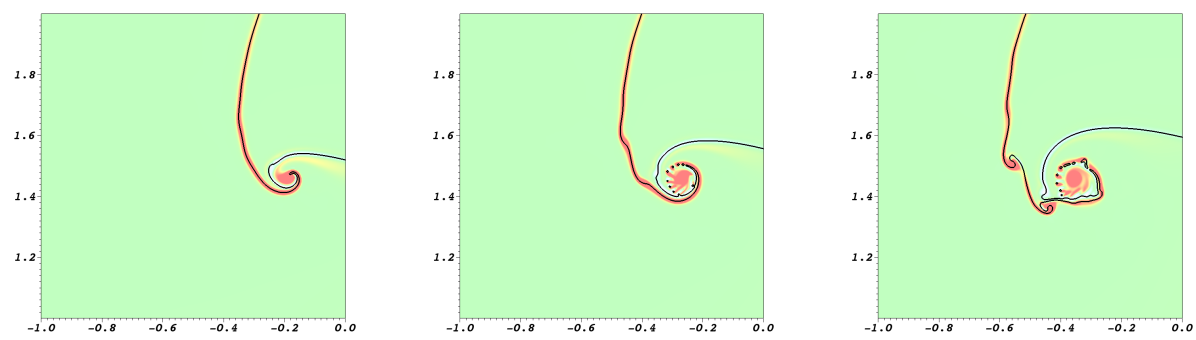

Figure 16: Problem $P_{2}$ for $r_{\rho}=5, R e=10^{4}$ and $a_{0}=1$ : Vorticity field and interface from left to right at times $t=2.4,2.6,2.8$. 
the action of the pre-existing vortex velocity field. The vortex layer increases in surface by stretching and at the same time, the stretching is due to generated vorticity layer which depends on the surface. This self-amplifying mechanism is not present when surface tension is only acting and is typical of an exponential increase. Hence it might explain why the exponential thinning of the tip evolution is observed. The triggering of an instability that becomes visible at later times might be also another mechanism that enhances such a thinning (figure 8). The temporal evolution of the total circulation in the regions of positive vorticity obtained from numerical simulations (Figure 15) reveal a significant increase on the vorticity production during the development of the interfacial instabilities observed in Figure 8, which occur to become visible for $t>20$ when $r_{\rho}=1.2$ and $t \gtrsim 15$ when $r_{\rho}=0.8$. The time separation between these two stages (tip dynamics and instability) depend on the Reynolds number. For $r_{\rho}>1$, the interface is Rayleigh-Taylor (RT) unstable on the external side due to the velocity field of the pre-existing vortex (for more on instability see section 6.1). In addition the vorticity generated at the interface during the initial instants leads to shear effects. The RT destabilization of the interface enhanced by the shear produced on this side, generates very thin sheets of heavier fluid (fluid 2) that are left behind. This effect strongly depends on the vorticity production rate and therefore on the density ratio. To understand the role of viscosity, let us compare the dynamics of the interface for $r_{\rho}=5$ and $a_{0}=1$ at Reynolds numbers $R e=10^{3}$ (figure 9) and $R e=10^{4}$ (figure 16). This shows that the tip dynamics is not modified but the instability period occurs faster for larger Reynolds. This effect explains the dependency of results with respect to the Reynolds number.

\subsection{Ligament dynamics and instability dynamics for $r_{\rho}<1$}

For $r_{\rho}<1$ the vorticity production changes sign in equation (20) with respect to case $r_{\rho}>1$. This is indeed observed in figure 8 . The change of vorticity sign induces a relative velocity (figure 17) which acts opposite to the preexisting vortex velocity field. The tip evolution is different : the ligament is thicker compared to the monophasic case. An instability is also present and occurs now on the internal side (figure 18): the RT instability is present only on this side. The appearance of instability is affected by the Reynolds number as discussed in section 6.1. 


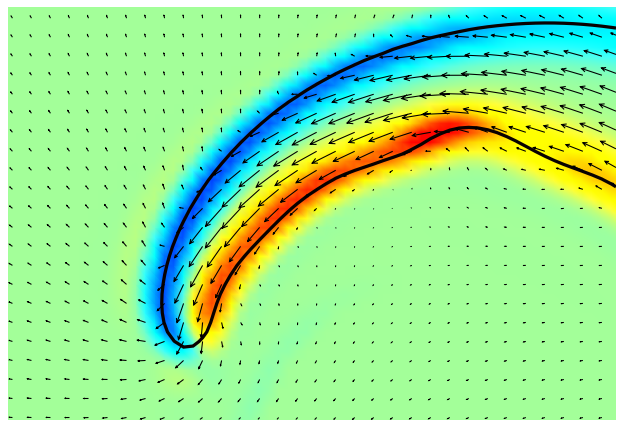

Figure 17: Problem $P_{2}$ for $r_{\rho}=0.2, R e=1000$ and $a_{0}=0.1$. Velocity field obtained using the filtered vorticity field at $t=2.4$. Color represents the vorticity field.
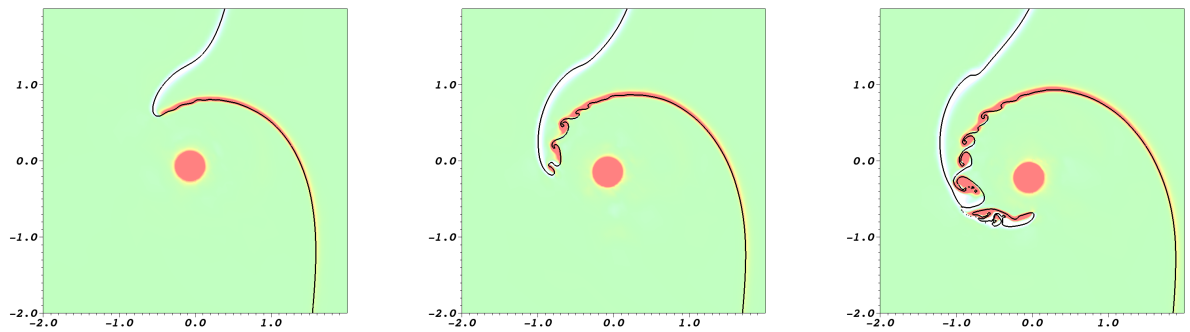

Figure 18: Problem $P_{2}$ for $r_{\rho}=0.2, a_{0}=0.1$ and $R e=2 \cdot 10^{3}$. Vorticity field and interface at times $t=1.5,2.0,2.5$. 

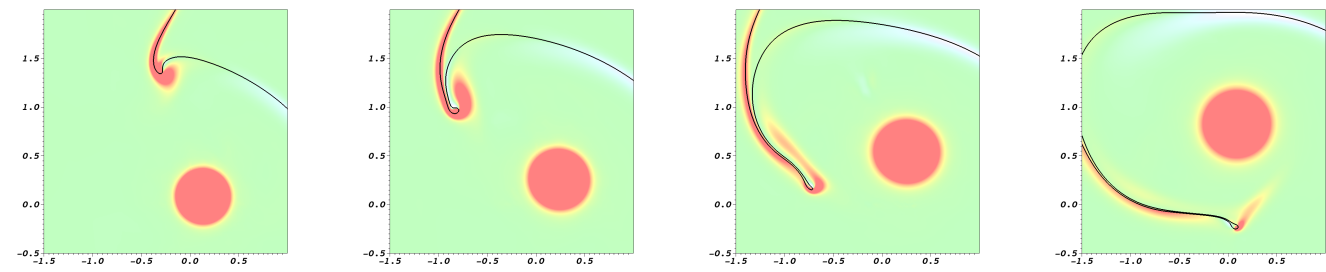

Figure 19: General problem for $r_{\rho}=5, W e=1000, R e=1000$ and $a_{0}=0.1$. Vorticity and Interface contours at $t=2,3,4,5$.

\section{General problem : density and surface tension effects together.}

We now consider cases in which both inertia $r_{\rho} \neq 1$ and surface tension (finite $W e)$ act as vorticity sources. Below we principally study flows with $r_{\rho}>1$. When both mechanisms are acting, a competition occurs between them: at a given $r_{\rho}>1$, the thinning due to density wins over the rim due to surface tension for high enough Weber numbers. For instance this occurs at $r_{\rho}=5$ and $W e=1000$ (figure 19). Very small structures are generated by inertial effects and the size of the rim, when present, decreases (figure 20 left). The vortex shed by the interface through baroclinic term is still present and is able to induce interface fragmentation. By further decreasing Weber number, surface tension dominates the dynamics creating a liquid rim at the tip of the ligament (figure 21) that grows after being formed (figure 20 right). A vortex is still shed from the interface but it is not strong enough to entrain rim and ligament, and is unable to desintegrate these structures. The rim structure is similar to the one observed in problem $P_{1}$.

One is able to define two critical Weber numbers $W e_{c}^{(1)}$ and $W e_{c}^{(2)}$. For $W e<W e_{c}^{(1)}$, the ligament does not appear while for $W e>W e_{c}^{(1)}$, the rim appears. By examining the rim size $h_{\max }(t)$ as a function of time (figure 22 left), one further distinguish two intervals: for $W e_{c}^{(1)}<W e<W e_{c}^{(2)}$, surface tension is sufficiently intense to form and stabilize a rim, $h_{\max }(t)$ growing in time as soon as a ligament is formed (see for example figure 20 right). When $W e>W e_{c}^{(2)}$, the rim exists but $h_{\max }(t)$ decreases in time leading to 

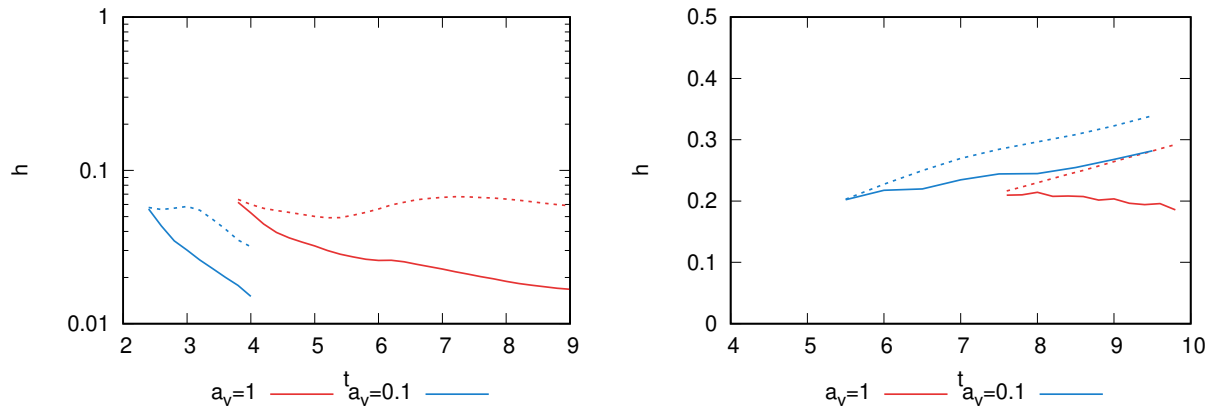

Figure 20: General problem for $r_{\rho}=5, R e=1000$ and (left) $W e=1000>W e_{c}^{(2)}$ and (right) $W e_{c}^{(1)}<W e=100<W e_{c}^{(2)}$. Temporal evolution of $h_{\text {min }}(t)$ (continuous line) and rim size $h_{\max }(t)$ (dashed line).
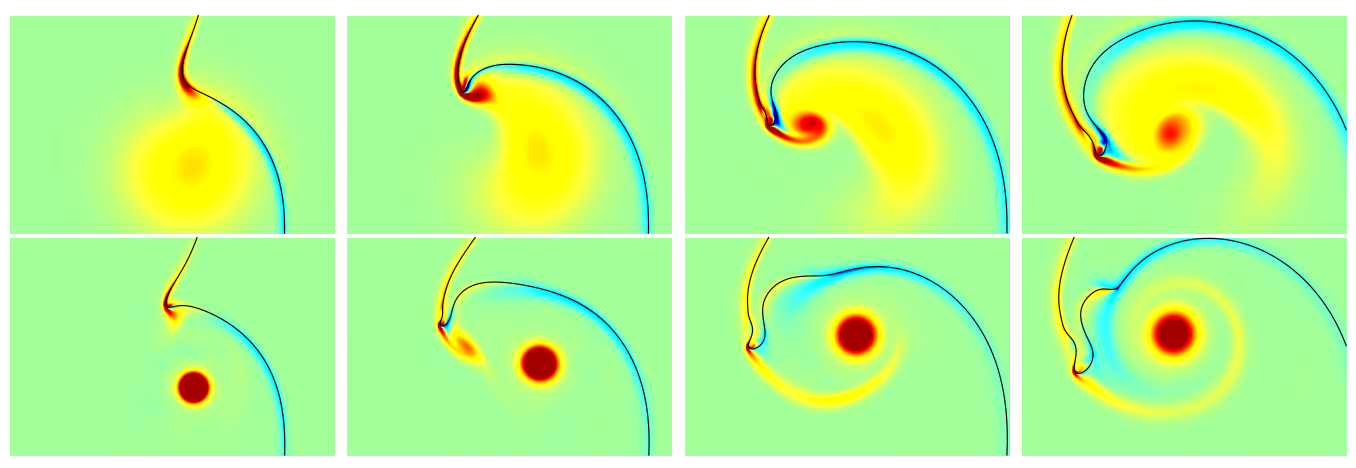

Figure 21: General problem for $r_{\rho}=5, W e=100, R e=1000$. (Top figures) $a_{0}=1$. (Bottom figures) $a_{0}=0.1$. Vorticity and interface contours at $t=2,4,6,8$.
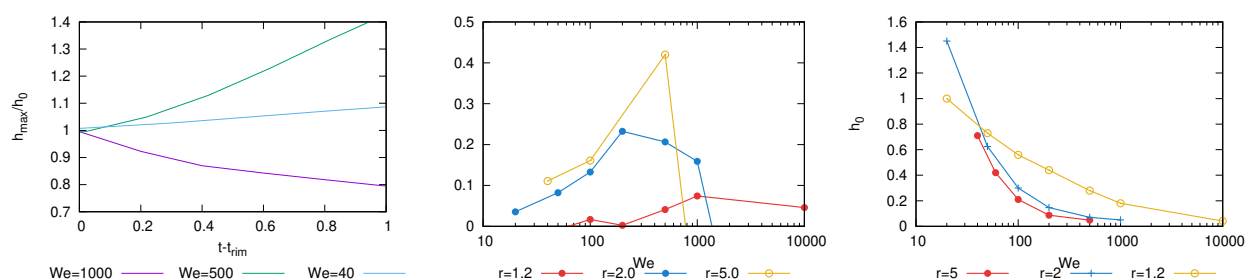

Figure 22: General problem $R e=1000, a_{0}=1$ and various Weber numbers $W e$. (Left) Temporal evolution of the rim size $h_{\max }(t)$ for $r_{\rho}=5$. (Middle) Growth-rate of the rim size $h_{\max }(t)$ as a function of $W e$ for $r_{\rho}=1.2,2,5$. (Right) Initial rim size $h_{0}$ as a function of $W e$ in the range $W e_{c}^{(1)}\left(r_{\rho}\right)<W e<W e_{c}^{(2)}\left(r_{\rho}\right)$. 


\section{NO RIM QUASI-STABLE UNSTABLE RIM RIM}

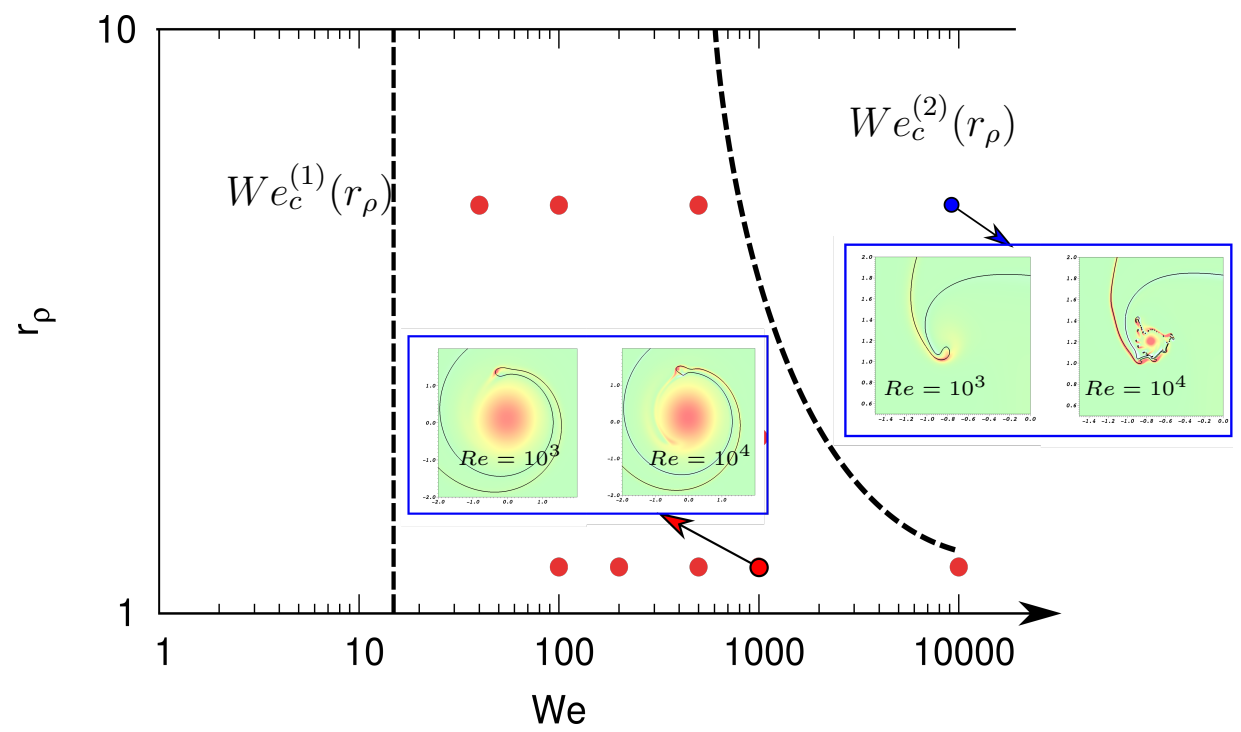

Figure 23: General problem for $R e=1000, a_{0}=1$. Regime map of conditions in the $\left(W e, r_{\rho}\right)$ plane upon which a stable structure at the ligament tip appears. The dashed line provides the numerically estimated values of $W e_{c}^{(2)}\left(r_{\rho}\right)$ and $W e_{c}^{(1)}\left(r_{\rho}\right)$. In the insets examples of the interface and vorticity snapshot for $R e=10^{3}$ and $R e=10^{4}$ in the two different regions identified. The snapshots correspond to $\mathrm{t}=15$ for the case $\left(W e, r_{\rho}\right)=$ $\left(10^{3}, 1.2\right)$ and $\mathrm{t}=4$ for $\left(W e, r_{\rho}\right)=\left(10^{4}, 5\right)$ 
the appearance of very small fragments difficult to resolve numerically (see for example figure 19). The critical value $W e_{c}^{(2)}\left(r_{\rho}\right)$ is eventually obtained as the value of $W e$ for which a sharp transition between negative or positive growth of the rim size $h_{\max }(t)$ occurs (figure 22 middle).

Figure 23 summarize all the simulations in a $W e-r_{\rho}$ map for which a rim forms and grows. The dashed curve displays $W e_{c}^{(1)}\left(r_{\rho}\right)$, value below which the rim does not appear. This curve indicates an only slight dependency if any on the density ratio. On the contrary, the value of $W e_{c}^{(2)}\left(r_{\rho}\right)$ strongly depends on the density ratio, tending to infinity as $r_{\rho} \rightarrow 1$. This threshold is $W e_{c}^{(2)} \approx 2000$ for $r_{\rho}=2$ and it is further reduced as the density ratio increases. Finally, a proxy for the size of structures generated in the regime $W e_{c}^{(1)}<W e<W e_{c}^{(2)}$ is provided by the values of $h_{0}$ (figure 22 right). Each curve begins near $W e_{c}^{(1)}\left(r_{\rho}\right)$ which depends only slightly on the density ratio. The last point of the each curve corresponds to $W e_{c}^{(2)}$ (except for $r_{\rho}=1.2$, where this value was not evaluated due to limited resolution). For all the points between $W e_{c}^{(1)}$ and $W e_{c}^{(2)}$, the initial rim size $h_{0}$ decreases as $W e$ and $r_{\rho}$ increase in this regime.

In order to understand the role of viscosity on the dynamics, the Reynolds number is varied though keeping the same dynamical viscosity for the two fluids. For a case within the region $W e_{c}^{(1)}<W e<W e_{c}^{(2)}\left(r_{\rho}\right)$ (density ratio $r_{\rho}=1.2$ and $\left.W e=1000\right)$ the vorticity generated by the interface does not significantly change the dynamics of the interface itself and the Reynolds number does not influence the interface shape significantly even for the relatively long times shown in the bottom inset of figure 23. For $W e>W e_{c}^{(2)}\left(r_{\rho}\right)$, the value Reynolds number influences interface position and vorticity field (for instance at $r_{\rho}=5$ and $W e=1000$ see the upper inset in figure 23). In this case, vorticity generated at the interface have an important effect on the interface itself, strongly coupling the dynamics of the interface with the flow changes induced by the presence of the interface.

For flows with $r_{\rho}<1$, the ligament opens up because of vorticity produced possesses an opposite effect of the pre-existing vortex. Surface tension $W e \neq \infty$ has a stabilizing effect on the instability at long times. Again the instability is more pronounced as Reynolds number is increased (figure 24). 

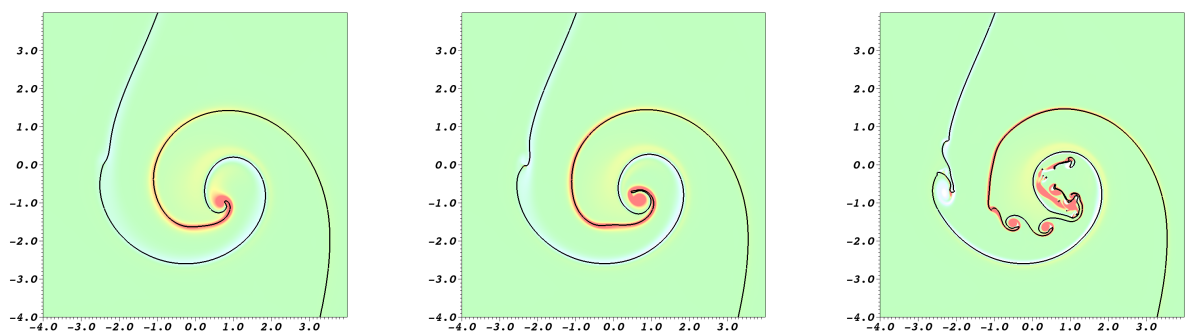

Figure 24: General problem A for $r_{\rho}=0.2, W e=1000$ and from (left) $R e=10^{3}$, (center) $2.10^{3}$, (right) $10^{4}$ : Vorticity field and interface at $t=10$.
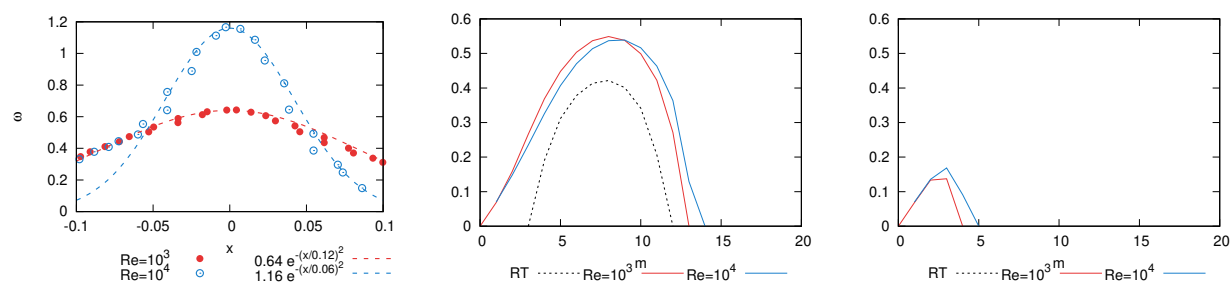

Figure 25: (Left) Vorticity field $\omega$ generated around the interface at $t=15$ obtained from numerical simulations for $r_{\rho}=1.2$ and $a_{0}=1.0$ and (red) $R e=10^{3}$ and (blue) $R e=10^{4}$. (Middle) Inviscid growth-rate $\varpi_{i}$ obtained from linear theory for $W e=1000$ in absence of the vorticity layer (dashed) or presence of a layer (red) $R e=10^{3}$ and (blue) $R e=10^{4}$. (Right) Same as before for $W e=100$.

\subsection{Modified Rayleigh-Taylor instability.}

The destabilization of the interface described before can be attributed to a combined Rayleigh-Taylor (RT) Kelvin-Helmholtz (KH) instability.

In order to assert how the RT destabilization is modified by the KH destabilization, a simplified analysis with one interface only is presented in appendix Appendix C. We discuss separately the results obtained in the quasi-stable regime described in figure $23\left(W e_{c}^{(1)}<W e<W e_{c}^{(2)}\left(r_{\rho}\right)\right)$, which is a common situation for fluids with small density ratios, and the fully unstable regime $\left(W e>W e_{c}^{(2)}\right)$ more common for large density ratios.

As an example of the classical situation found in the $W e_{c}^{(1)}<W e<$ $W e_{c}^{(2)}\left(r_{\rho}\right)$ regime, we discuss the case of $r_{\rho}=1.2, W e=\infty$ and two different 

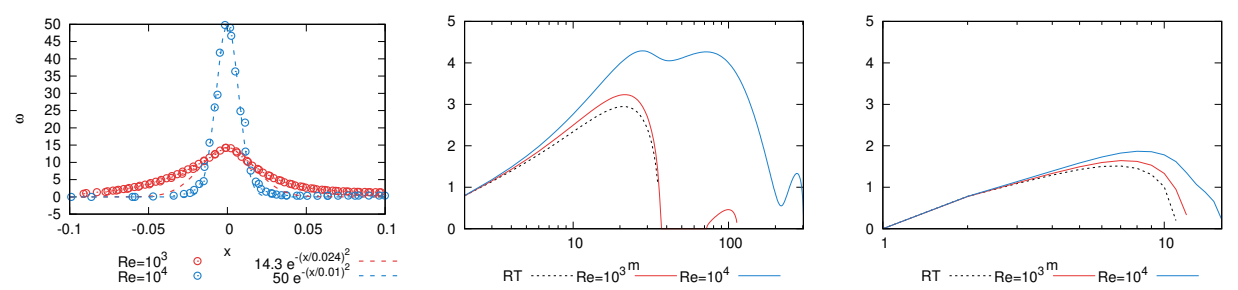

Figure 26: (Left) Vorticity field $\omega$ generated around the interface at $t=2.2$ for the simulation with $r_{\rho}=5.0, a_{0}=1.0, W e=\infty$, and (red) $R e=10^{3}$ and (blue) $R e=10^{4}$. (Middle) Inviscid growth-rate $\varpi_{i}$ for $W e=1000$ in absence of the vorticity layer (dashed) or presence of a layer corresponding to the base flow observed for a thickness corresponding to (red) $R e=10^{3}$ and (blue) $R e=10^{4}$. (Right) Same as before for $W e=100$.

Reynolds numbers. Figure 25(left) depicts vorticity generated around the interface extracted from numerical simulations at $t=15$ (before the instability becomes visible in time on figure 8). Profiles are well fitted by gaussian distributions around the interface where the thickness of the vorticity layer approximately corresponds to $\delta \approx \sqrt{t / R e}$. Note that circulation $\Gamma \approx \omega \delta$ remains approximately similar for the two Reynolds numbers, indicating that vorticity production is not much influenced by viscosity. The results of the simplified stability problem (middle and right plots in figure 25) show that RT instability without shear is present for $W e \leq 1000$,but completely suppressed for $W e=100$. The presence of the vorticity layers leads to an additional destabilization mechanism of the interface leading positive grow-rates even for $W e=100$. The growth rates remain relatively small and neither the most unstable wavelengths nor the magnitude of those are sensitive to Reynolds number tested here. This result is therefore consistent with the slight dependence of the interfacial structures when varying the Reynolds number for small the density ratios even for large Weber numbers (see for example the bottom inset in figure 23).

For larger density ratios $\left(r_{\rho}=5\right)$, the vorticity layer obtained instants before the instability becomes visible (figure 26 left) is such that its circulation is similar for the simulations obtained at two different Reynolds number while thickness is still relatively well approximated by $\delta \approx \sqrt{t / R e}$. Unlike the case $r_{\rho}=1.2$, the inviscid stability code results obtained for $W e=1000>W e_{c}^{(2)}$ (figure 26middle) differs a strong Reynolds number dependency on the un- 
stable range by the combined effects of the RT and KH instability. This result is consistent with the observations from the inset of Figure 23 for $\left(W e, r_{\rho}\right)=\left(10^{4}, 5\right)$, where the interface topology is strongly influenced by the Reynolds number. Note that for $r_{\rho}=5$ if the value of the Weber number is decreased below the critical threshold ( $W e_{c}^{(1)}<W e<W e_{c}^{(2)}$ ) the flow is still unstable but the effect of the $\mathrm{KH}$ is only secondary and the most unstable wavelength and the growthrates are governed by the pure RT instability and therefore there is only a minor dependence of the interfacial topology changes on the Reynolds number as discussed previously (results for $W e=100$ shown in Figure 26right).

The above results indicate that the regimes identified in figure 23 correspond to situations in which the RT instability is affected or not by the $\mathrm{KH}$ instability: for $W e>W e_{c}^{(2)}$ the $\mathrm{KH}$ plays a major role on the destabilization of the surface and therefore the presence of a vorticity layer modify the interfacial patterns observed, for $W e<W e_{c}^{(2)}$ RT prevails, the Reynolds number playing only a marginal effect on the interface evolution.

\section{Conclusions}

This work focuses on the basic interactions between a vortex and an interface separating two incompressible fluids. Namely, how vorticity produced at an interface influences the dynamics of this very same interface and how this generation produces small scales at the origin of small droplets of fluid. Depending on the main mechanism (surface tension or density effect or both), one observes 1) a change from algebraic to exponential in the ligament width decrease 2) the role of Reynolds number 3) the interaction between small scale produced and the large scale. When the Weber number is less than a critical Weber $W e_{c}^{(1)} \approx 14$, topological changes of the interface are not significantly modified by the vorticity produced at this interface. This remains valid irrespective of density ratio and Reynolds number. By contrast, when the Weber number is greater than a critical Weber $W e_{c}^{(2)}$, the interface is strongly influenced by the vorticity production and the combined effect of Rayleigh-Taylor and Kelvin-Helmholtz instabilities appear to have a major impact on the appearance of small wavelengths. In this regime density ratio and Reynolds number controling the generation of small structures. In 
the Weber number interval $W e \in\left[W e_{c}^{(1)}, W e_{c}^{(2)}\right]$, a transition regime appears where destabilization of the interface is basically controlled by a pure Rayleigh-Taylor instability and vorticity sheets generated by the interface, while influencing the interface response and the characteristic size of the structures, do not depend significantly on the Reynolds number. The range of Weber numbers for which the interval $\left[W e_{c}^{(1)}, W e_{c}^{(2)}\right]$ is visible, vanishes as the density ratio increases. These results are applicable to understand the dynamics of two-dimensional structures even in three-dimensional flows, although certainly more work will be required in the future to completely characterize the full three-dimensional dynamic interaction between vorticity and interfaces.

[1] C.-S. Yih, Instability due to viscosity stratification, Journal of Fluid Mechanics 27 (2) (1967) 337-352.

[2] A. Hooper, W. Boyd, Shear-flow instability at the interface between two viscous fluids, Journal of Fluid Mechanics 128 (1983) 507-528.

[3] E. J. Hinch, A note on the mechanism of the instability at the interface between two shearing fluids, Journal of Fluid Mechanics 144 (1984) 463465.

[4] D. H. Sharp, Overview of rayleigh-taylor instability, Tech. rep., Los Alamos National Lab., NM (USA) (1983).

[5] E. Villermaux, C. Clanet, Life of a flapping liquid sheet, Journal of fluid mechanics 462 (2002) 341.

[6] Y. Ling, D. Fuster, T. G., S. Zaleski, A two-phase mixing layer between parallel gas and liquid streams: multiphase turbulence statistics and influence of interfacial instability, Journal of Fluid Mechanics 859 (2019) 268-307.

[7] S. Ohring, H. J. Lugt, Interaction of a viscous vortex pair with a free surface, Journal of Fluid Mechanics 227 (1991) 47-70.

[8] H. J. Lugt, S. Ohring, The oblique ascent of a viscous vortex pair toward a free surface, Journal of Fluid Mechanics 236 (1992) 461-476. 
[9] M. S. Longuet-Higgins, Mass transport in water waves, Philosophical Transactions of the Royal Society of London. Series A, Mathematical and Physical Sciences 245 (903) (1953) 535-581.

[10] M. S. Longuet-Higgins, Capillary rollers and bores, Journal of Fluid Mechanics 240 (1992) 659-679.

[11] T. Lundgren, P. Koumoutsakos, On the generation of vorticity at a free surface, Journal of Fluid Mechanics 382 (1999) 351-366.

[12] J.-Z. Wu, A theory of three-dimensional interfacial vorticity dynamics, Physics of Fluids 7 (10) (1995) 2375-2395.

[13] J. Wu, J. Wu, Boundary vorticity dynamics since lighthill's 1963 article: review and development, Theoretical and computational fluid dynamics 10 (1-4) (1998) 459-474.

[14] M. Brøns, M. C. Thompson, T. Leweke, K. Hourigan, Vorticity generation and conservation for two-dimensional interfaces and boundaries, Journal of Fluid Mechanics 758 (2014) 63-93.

[15] S. Terrington, K. Hourigan, M. Thompson, The generation and conservation of vorticity: deforming interfaces and boundaries in twodimensional flows, Journal of Fluid Mechanics 890.

[16] R. Cresswell, B. Morton, Drop-formed vortex rings. the generation of vorticity, Physics of Fluids 7 (6) (1995) 1363-1370.

[17] M. A. André, P. M. Bardet, Interfacial shear stress measurement using high spatial resolution multiphase piv, Experiments in Fluids 56 (6) (2015) 132 .

[18] A. Iafrati, E. Campana, Free-surface fluctuations behind microbreakers: space-time behaviour and subsurface flow field, Journal of Fluid Mechanics 529 (2005) 311.

[19] A. Iafrati, A. Babanin, M. Onorato, Modeling of ocean-atmosphere interaction phenomena during the breaking of modulated wave trains, Journal of Computational Physics 271 (2014) 151-171.

[20] L. Deike, S. Popinet, W. K. Melville, Capillary effects on wave breaking, Journal of Fluid Mechanics 769 (2015) 541-569. 
[21] L. Deike, W. K. Melville, S. Popinet, Air entrainment and bubble statistics in breaking waves, Journal of Fluid Mechanics 801 (2016) 91-129.

[22] A. Loisy, A. Naso, Interaction between a large buoyant bubble and turbulence, Physical Review Fluids 2 (1) (2017) 014606.

[23] B. Zhang, Y. Ling, P.-H. Tsai, A.-B. Wang, S. Popinet, S. Zaleski, Short-term oscillation and falling dynamics for a water drop dripping in quiescent air, Physical Review Fluids 4 (12) (2019) 123604.

[24] J. Hoepffner, G. Paré, Recoil of a liquid filament: escape from pinch-off through creation of a vortex ring, Journal of fluid mechanics 734 (2013) $183-197$.

[25] S. Quan, D. P. Schmidt, J. Hua, J. Lou, A numerical study of the relaxation and breakup of an elongated drop in a viscous liquid, Journal of fluid mechanics 640 (2009) 235-264.

[26] X. Guo, L. Shen, Interaction of a deformable free surface with statistically steady homogeneous turbulence, Journal of fluid mechanics 658 (2010) 33-62.

[27] M. Rossi, D. Fuster, Vorticity production at fluid interfaces in twodimensional flows, arXiv preprint arXiv:2102.05878.

[28] S. Popinet, A quadtree-adaptive multigrid solver for the Serre-GreenNaghdi equations, Journal of Computational Physics 302 (2015) 336358.

[29] D. Fuster, A. Bagué, T. Boeck, L. Moyne, A. Leboissetier, S. Popinet, P. Ray, R. Scardovelli, S. Zaleski, Simulation of primary atomization with an octree adaptive mesh refinement and vof method, International Journal of Multiphase Flow 35 (6) (2009) 550 - 565.

[30] D. Fuster, G. Agbaglah, C. Josserand, S. Popinet, S. Zaleski, Numerical simulation of droplets, bubbles and waves: state of the art, Fluid dynamics research 41 (6) (2009) 065001.

[31] S. Popinet, An accurate adaptive solver for surface-tension-driven interfacial flows, Journal of Computational Physics 228 (16) (2009) 58385866. 
[32] S. Popinet, Numerical models of surface tension, Annual Review of Fluid Mechanics 50 (2018) 49-75.

[33] G. D. Weymouth, D. K.-P. Yue, Conservative volume-of-fluid method for free-surface simulations on cartesian-grids, Journal of Computational Physics 229 (8) (2010) 2853-2865.

\section{Appendix A. Passive spiral and test of curvature algorithm.}

In the classical monophasic case $\left(r_{\rho}=1\right.$ and $\left.W e=\infty\right)$, there is no vorticity source $(\Sigma=0)$ and the vortex evolves as a Lamb-Oseen vortex with an unsteady core size

$$
u_{\theta}=\frac{1-\exp \left(-\left(r / a_{v}\right)^{2}\right)}{r}, \quad \omega_{z}=\frac{2}{a_{v}^{2}} \exp \left(-\left(r / a_{v}\right)^{2}\right), \quad a_{v}^{2}(t)=a_{0}^{2}+\frac{4}{R e} t .
$$

In addition, the interface is passive and forms a ligament that rolls up in time (figure A.27). It becomes a spiral which can be expressed in polar coordinates $(r, \theta)$ as

$r\left(\phi_{0}, t\right)=\frac{1}{\cos \phi_{0}}, \quad \theta\left(\phi_{0}, t\right)=\frac{1}{r^{2}}\left[1-\exp \left(-\frac{r^{2}}{a_{v}^{2}(t)}\right)\right] t+\phi_{0} \quad$ with $\left.\phi_{0} \in\right]-\pi / 2, \pi / 2[$

with $a_{v}^{2}(t)=1+\frac{4}{R e} t$. Here below the viscous diffusion term $\frac{4}{R e} t$ is discarded. This analytical shape allows us to obtain quantities such as curvature and ligament size as a function of time. At time $t$, the curvilinear coordinate can be expressed using the integral

$$
s=\int_{-\pi / 2}^{\phi_{0}} \frac{\partial s}{\partial \phi_{0}} d \phi_{0} \quad \text { with } \frac{\partial s}{\partial \phi_{0}}=\sqrt{\left[\frac{\partial r}{\partial \phi_{0}}\right]^{2}+r^{2}\left[\frac{\partial \theta}{\partial \phi_{0}}\right]^{2}},
$$

The curvature $\kappa$ along this passive spiral is given by

$$
\kappa=\frac{r^{2} \theta^{\prime 3}+r r^{\prime} \theta^{\prime \prime}-r r^{\prime \prime} \theta^{\prime}+2 r^{\prime 2} \theta^{\prime}}{\left(r^{\prime 2}+r^{2} \theta^{\prime 2}\right)^{3 / 2}}, \quad r^{\prime} \equiv \frac{\partial r}{\partial \phi_{0}}, \quad \theta^{\prime} \equiv \frac{\partial \theta}{\partial \phi_{0}}
$$

where

$$
r^{\prime}=\frac{\sin \phi_{0}}{\left(\cos \phi_{0}\right)^{2}}, \quad \theta^{\prime}=1+t f(r) r^{\prime}, \quad f(r, t)=\frac{-2}{r^{3}}+2\left[\frac{1}{r^{3}}+\frac{1}{a_{0}^{2} r}\right] \exp \left(-\frac{r^{2}}{a_{0}^{2}}\right)
$$



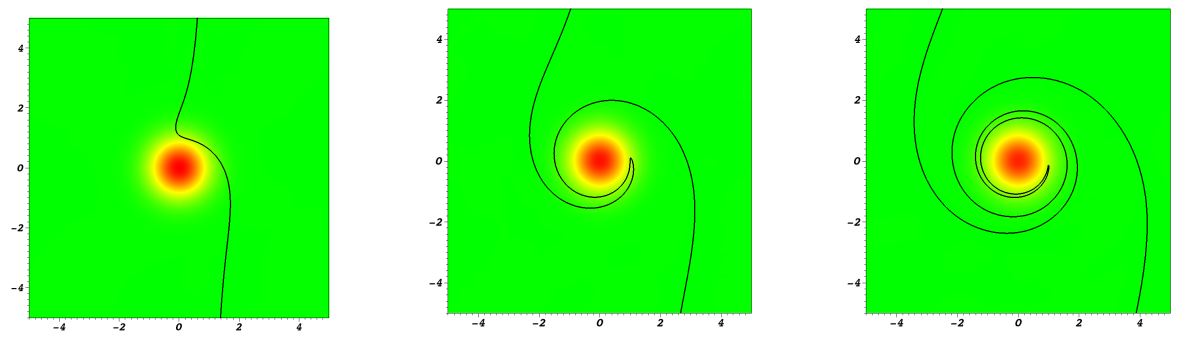

Figure A.27: The monophasic case (case $W e=\infty, r_{\rho}=1$ ) with passive tracer and $R e=1000$. From left to right. Vorticity field and interface evolution at $t=2.5,5,20$.

Theoretical expression (A.4) provides curvature as a function of $\phi_{0}$ and thus provides a point of comparison with the numerical simulations. Such a comparison tests the algorithm of Basilisk that evaluates curvature along the curve for a given time (see figure A.28a): curvature obtained from numerical simulations are in good agreement with these analytic findings whenever the curvature is below 20 . This value is controlled by the minimum grid size. Looking at the expression for $\kappa$ one may also guess that the maximum curvature occurs at a point for which $\theta^{\prime 2} \rightarrow 0$. Indeed for such a point, $\kappa \sim \frac{r r^{\prime} \theta^{\prime \prime}}{\left(r^{\prime 2}\right)^{3 / 2}}$. When in addition $r^{\prime} \rightarrow 0$ then the curvature can become large. This occurs at large time close to the point $\phi_{0}=0$ more precisely such as

$$
\phi_{0} \sim-1 /(t f(1)) \sim 1.89 / t \quad \text { since } f(1) \sim-0.53
$$

At this point $r^{\prime} \sim-\frac{1}{t f(1)}$ and $\theta^{\prime \prime} \sim t f(1)$ so that

$$
\kappa \sim-f^{3}(1) t^{3} \sim 0.15 t^{3}
$$

This theoretical results indicate the development of a tip that indefinitely increase its curvature: the variation evolves with $t^{3}$ for large times. By consequence, the ligament width evolves with $t^{-3}$ but no breakup of the ligament is observed in figure A.27. A numerical breakup can still be observed when the ligament reaches the size of the numerical cell. Figure A.28b shows the temporal evolution of maximal curvature obtained analytically and numerically as well as the evolution of the position in term of $\phi_{0}$ of the point of maximum curvature. Numerically $\phi_{0} t$ tends to a value close to 1.87 for large times and maximum curvature obtained from the numerical 

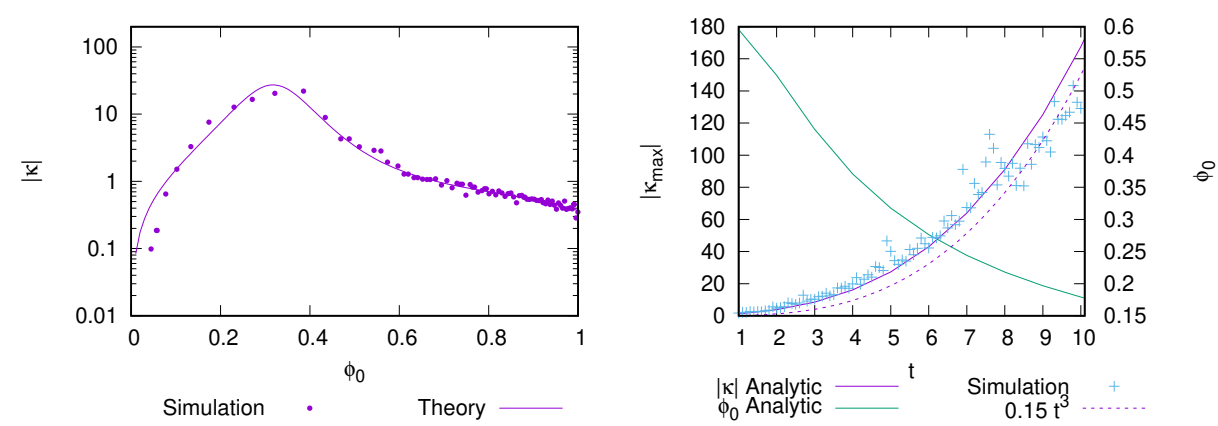

Figure A.28: Passive spiral case. (a) Curvature $|\kappa|$ as a function of $\phi_{0}>0$ at time $t=5$. Numerical values (dots) and theoretical values (line). (b) Maximum curvature $\left|\kappa_{\max }(t)\right|$ as a function of time. Crosses indicate the value obtained by numerical simulation and the toolkit performed using the Basilisk solver purple line corresponds to the analytic computation. The green line traces the corresponding value of $\phi_{0}(t)$.

simulations are in good agreement with analytic findings at large times. The above quantities are useful in order to compute an approximation of the source term $\Sigma$. Namely one assumes that the spiral is close to the passive case and uses equation (18) in the presence of surface tension only, or else expression (21) in presence of density difference only.

\section{Appendix B. Computation of curvature $\kappa$, ligament width $h_{\text {min }}$ and rim size $h_{\max }$}

At a given time, we numerically compute the maximum curvature $\left|\kappa_{\max }(t)\right|$ of a ligament, its width $h_{\min }(t)$ and when it exists the size $h_{\max }(t)$ of a rim. This appendix presents the algorithm that performs this task. It is adapted for the geometry observed our flow problem and will be used to investigate the influence of density only or of density and surface tension.

The curvature computation and its maximum over the interface are performed by a Basilisk tool. We have tested it on the passive spiral, where the analytical solution is known (see appendix Appendix A for details). Let us now explain how the ligament width $h_{\min }(t)$ is computed. Consider at a given time, an interface which is oriented from bottom towards top with increasing $s$ (this is due to the choice of orientation defined in section 2 

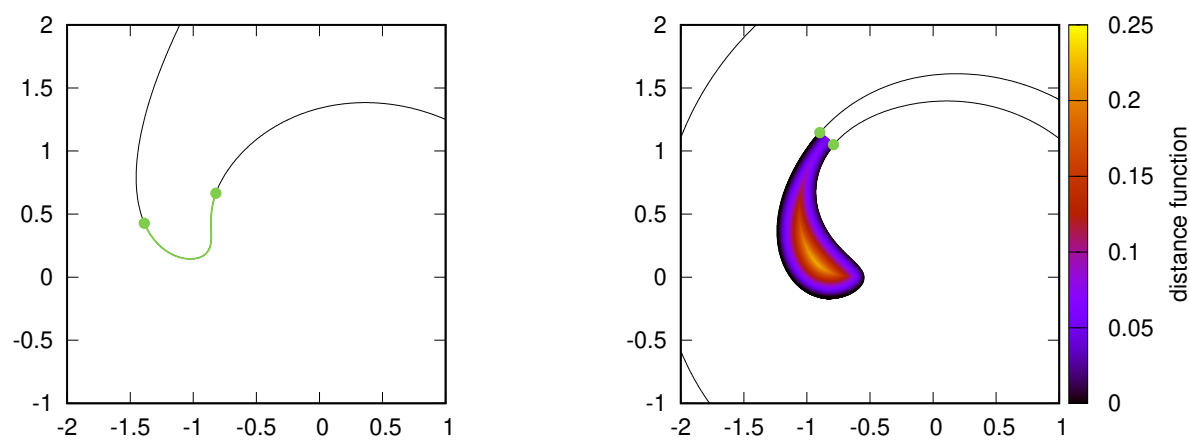

Figure B.29: Result of the algorithm that detects $h_{\min }$ (left) when no rim is formed and (right) when a rim is formed. In the latter case, the closest distance of a point of the rim to the interface is displayed as a color code. $h_{\max }$ is obtained from the maximum of the distance to the interface in the points defining the ligament region.

where interface $(I)$ is oriented by the tangent unit vector $\vec{t}=\vec{e}_{z} \times \vec{n}$ and $\vec{n}$ directed from phase 1 to 2$)$. We compute for any point $M_{1}\left(s_{1}\right)$ of this interface, the distance $D\left(M_{1}, M_{2}\right)$ from any other points $M_{2}\left(s_{2}\right)$ with $s_{2}<s_{1}$ on the interface. By decreasing $s_{2}$ starting from $s_{1}$, distance $D\left(M_{1}, M_{2}\right)$ first increases from zero. It may then continue increasing, or else may decrease before increasing again. In the second case, there exists a point $M_{3}$ which is associated to the first minimum distance of point $M_{1}$, one then defines $d\left(M_{1}\right) \equiv D\left(M_{1}, M_{3}\right)$. For some interface geometry, there is no point $M_{3}$ for any $M_{1}$ on the interface and we cannot define $d\left(M_{1}\right)$ (e.g. the interface at $\mathrm{t}=0)$. However when $d\left(M_{1}\right)$ can be defined for a certain interval of points $M_{1}$, the minimum minimorum of $d\left(M_{1}\right)$ gives the width of the ligament: this is precisely $h_{\text {min }}(t)$. The point $M_{\min }$ is associated to $h_{\min }$ and $M_{\text {comp }}$ is the companion point such that $h_{\min }=D\left(M_{\min }, M_{\text {comp }}\right)$. This is shown on figure B.29a at the extreme positions of the green interval.

When a rim appears on the ligament, this means that a constriction of the ligament is followed by an enlargement (figure B.29b). In order to get the size of the rim, one first defines the area $A_{\text {rim }}$ which is enclosed by a curve formed by the interface ( $I$ ) between points $M_{\min }$ and $M_{\text {comp }}$ together with the straight line joining $M_{\min }$ to $M_{\text {comp }}$. Thereafter one determines the point inside this area which is located at the maximal distance from the curve: Twice this distance provides the size of the rim $h_{\max }$. When $h_{\max }(t)=$ $h_{\text {min }}(t)$ there exists no rim (see figure 1 at $t=5$ ) and for $h_{\max }(t)>h_{\min }(t)$ 
there is a rim of size $h_{\max }(t)$ (figure 1 at $t=30$ top and middle ). The time at which this latter inequality first occurs defines $t_{\text {rim }}$ and

$$
h_{0} \equiv h_{\min }\left(t_{\text {rim }}\right)
$$

provides the size of the rim at the time of its appearance.

\section{Appendix C. Circular layer instability.}

We study the inviscid instability of the base flow $\left(0, U_{\theta}^{(0)}(r), 0\right)$ formed by a Rankine vortex of circulation $\Gamma_{0}$ and size $a_{0}$ surrounded by an axisymmetric interface $r=d_{0}$ separating fluid denoted by subscript in located in $r<d_{0}$ from a different fluid denoted by subscript out located in $r>d_{0}$ and characterized by a surface tension $\sigma$. On this interface, a vorticity layer of constant amplitude $\omega_{L}$ and of width $\delta d_{0}$ can be added or not. The velocity $U_{\theta}^{(0)}(r)$ is assumed to remain continuous across the interface. We use dimensionless quantities based on $d_{0}$ and $\Gamma_{0} /(2 \pi)$ and introduces the Atwood number $A_{t w}=\left(\rho_{\text {out }}-\rho_{\text {in }}\right) /\left(\rho_{\text {out }}+\rho_{\text {in }}\right)$. Two-dimensional infinitesimal perturbations of such a base flow are governed by linearized Euler equations. One uses cylindrical coordinates and obtains in a standard manner, an analytical implicit equation relating the complex pulsation $\varpi(m)$ of the normal mode $\Phi(r) \exp (i(m \theta-\varpi(m) t)$ of azimuthal wavenumber $m$. We are not reproducing these standard computations. The two situations $A_{t w}>0$ and $A_{t w}<0$ are then analyzed. For $a_{0} / d_{0}=1$, the Rankine vortex is touching the interface and the complex pulsation $\varpi$ is indeed explicit:

$\varpi=\frac{m}{|m|}\left(|m|-\frac{1}{2}\left(1-A_{t w}\right) \pm \sqrt{\frac{1}{4}\left(1-A_{t w}\right)^{2}+|m| A_{t w}+\frac{\left(m^{2}-1\right)|m|}{2 W e}}\right)$

with $n=|m|$. For $A_{t w}>0$, the base flow is stable since the growth rate i.e. the imaginary part $\varpi_{i}$ of pulsation $\varpi$ remains zero. For $A_{t w}<0$, the Rankine vortex with an interface is Rayleigh-Taylor unstable (see figure C.30 for $A_{t w}=-0.66$ and $a_{0}=1$ ). For $A_{t w}=-0.66$ and a smaller core size $a_{0} / d 0<1$, the interface tends to destabilize faster (figure C.31 left). 


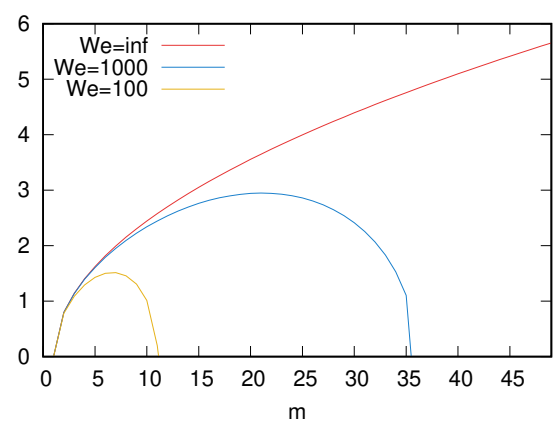

Figure C.30: Growth rate $\varpi_{i}$ as a function of wavenumber $m$ for $A_{t w}=-0.66, a_{0}=1$ and various Weber numbers $W e$.
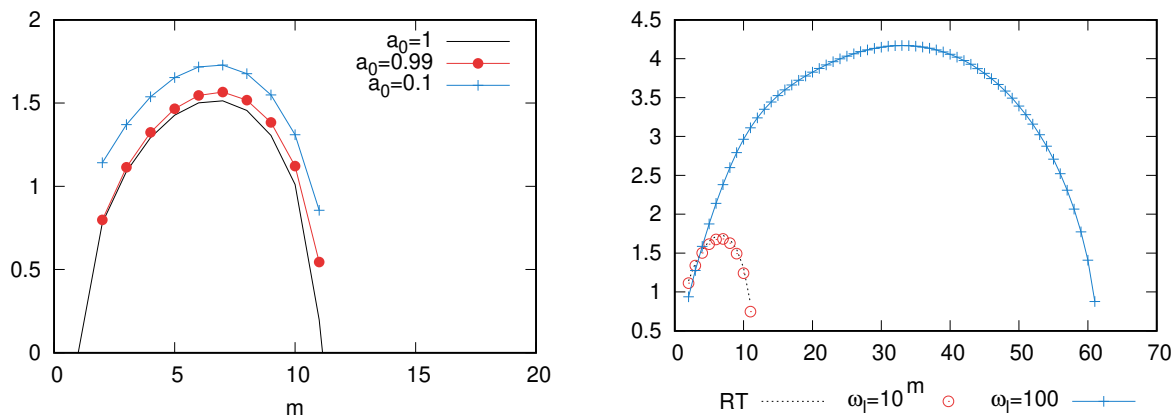

Figure C.31: Growth rate $\varpi_{i}$ as a function of wavenumber $m$ for $A_{t w}=-0.66$ and $W e=100$. (Left) Influence on the pure Rayleigh-Taylor instability of core size $a_{0} / d_{0}$ (Right) Influence of a vortex layer on the instability for $\delta=0.01$ with $\omega_{L}=0$ (dots), $\omega_{L}=10$ (red circles) and $\omega_{L}=100$ (blue crosses). 
Let us add a vorticity layer of constant amplitude $\omega_{L}$. For $A_{t w}<0$, this layer is located at $1<r<(1+\delta)$, for $A_{t w}>0$, the layer is located at $(1-\delta)<$ $r<1$. Such an axisymmetric vortex layer is known to be unstable in a monophasic case giving rise to a two-dimensional instability wave. When the flow is RT unstable $\left(A_{t w}<0\right)$, the case $\omega_{L}=10$ and $\delta=0.01$, is very similar to the pure RT case. Reversely for $\omega_{L}=100$ and $\delta=0.01$, the situation is quite different (see figure C.31right). In the particular case $A_{t w}=-0.66$ and $W e=100$, when the shear stress is not sufficient $\left(\omega_{L}=10\right.$ and $\left.\delta=0.01\right)$, the $\mathrm{KH}$ instability does not play a significant role on the obtained growth-rates. When $\omega_{L}=100$ and $\delta=0.01$, the KH instability plays a role on the range of wavelengths that become unstable, significantly increasing the growth-rate. The cases corresponding to the numerical simulations are presented inside the main text. 